Ann. Sci. forest., 1972, 29 (1), 3-34.

\title{
CONTRIBUTION A L'ÉTUDE DE LA NUTRITION ET DE LA FERTILISATION DES PLANTATIONS D'ÉPICÉA COMMUN DANS LE MASSIF CENTRAL
}

\author{
M. BONNEAU
}

avec la collaboration technique de M. AdrIAN, Maryse BrTsCH, Françoise Georges

Station de Recherches sur les Sols forestiers et la Fertilisation, Centre national de Recherches forestières, I.N.R.A., 54 - Champenoux, par Einville

\begin{abstract}
RÉSUMÉ
Des essais de fertilisation et des enquêtes de nutrition montrent que les jeunes plantations d'Épicéa sont fortement carencées en azote dans les stations d'altitude du Massif Central et carencées en phosphore dans les landes à callune de moyenne altitude. Dans les premières, une fertilisation calcique copieuse apporte une amélioration de la croissance assez importante, mais ne relève pas la teneur des aiguilles en azote; une fertilisation azotée complémentaire augmente encore davantage, mais de maniere temporaire, I 'accroissement annuel en hauteur. Dans les secondes, une fertilisation phosphatée au moment de la plantation améliore considérablement la croissance juvénile. Dans les landes à fougère de moyenne altitude, la nutrition est en général satisfaisante; une fertilisation phosphatée peut être bénéfique dans quelques cas particuliers.
\end{abstract}

\section{INTRODUCTION}

Le Massif Central est, à cause de la faible valeur agricole de ses terroirs, de son climat humide, de ses sols à texture légère, la première région de France pour le reboisement en résineux. L'Épicéa commun (Picea abies) est l'essence la plus employée et sa réussite est en général satisfaisante. L'ampleur de son utilisation rendait cependant légitimes des recherches sur sa nutrition et sa fertilisation, soit dans les plantations, soit dans les peuplements adultes. C'est pourquoi nous avons entrepris, depuis 10 ans, des expérimentations et des études sur les jeunes plantations; malgré le caractère provisoire de certains résultats, nous pensons utile de les mettre à la disposition des praticiens. 
Nous présenterons d'abord les essais de fertilisation en les classant sous deux rubriques : ceux de haute-altitude et ceux de moyenne altitude, puis nous verrons dans quelle mesure on peut généraliser les résultats des essais de moyenne altitude, les plus intéressants probablement pour la pratique, à la lumière d'une enquête plus générale sur la nutrition des plantations dans l'Ouest du Massif Central.

\section{I. - LES EXPÉRIMENTATIONS A HAUTE ALTITUDE}

\section{1. - Essai du Lac de Guéry}

Il a déjà été rendu compte des premiers résultats de cet essai (BONNEAU, 1965).

\section{a) Conditions de station et implantation de l'essai}

Bien qu'elles aient déjả été partiellement décrites dans l'article ci-dessus, nous rappellerons brièvement les conditions de station. Il s'agit d'un sol d'altitude $(1320 \mathrm{~m})$, sur mélange de basalte et d'andésite, très riche en humus, à $\mathrm{C} / \mathrm{N}$ bas, avec végétation de graminées mélangées d'un peu de Vaccinium myrtillus et $V$. Vitis idora. Si l'on se fie aux analyses habituelles, ce sol est bien pourvu en $\mathrm{P}_{2} \mathrm{O}_{5}$ assimilable ${ }^{1}(0,48$ a $1,29 \%$ suivant les horizons), de fertilité moyenne en $\mathrm{K}$ échangeable $(0,13$ à $0,14 \mathrm{~m}$.e. $/ 100 \mathrm{~g})$, très acide $(S / T$ de $2,5 \%$ en surface), Bien que ce sol ait probablement des caractères andiques, nous n'avions pas trouvé en 1965 de signes de toxicité aluminique.

Le dispositif, très simple, mis en place en 1961, comportait 4 répétitions en blocs complets et 4 traitements appliqués après un travail du sol par décapage et sous-solage :

- traitement $n^{\circ} l:$ témoin.

- traitement $n^{\circ} 2$ : PKCa surface : mélange sur $10 \mathrm{~cm}$ de profondeur, à l'emplacement futur des plants, de $80 \mathrm{~g}$ d'hyperphosphate potassique $20-10$ ( $16 \mathrm{~g}$ de $\mathrm{P}_{2} \mathrm{O}_{5}, 8 \mathrm{~g}$ de $\mathrm{K}_{2} \mathrm{O}$ et $30 \mathrm{~g}$ de $\mathrm{CaO}$ ). Ce premier apport a été complété en 1966 par l'épandage superficiel, sur les bandes décapées ( $50 \mathrm{~cm}$ de large environ), de $50 \mathrm{~g}$ d'hyperphosphate et $20 \mathrm{~g}$ de sulfate de potassium par mètre linéaire de bande, soit $75 \mathrm{~kg}$ de $\mathrm{P}_{2} \mathrm{O}_{5}, 50 \mathrm{~kg}$ de $\mathrm{K}_{2} \mathrm{O}$ et $150 \mathrm{~kg} \mathrm{de} \mathrm{CaO}$ par hectare concentrés sur les lignes de plants.

- traitement $n^{0} 3:$ NPKCa surface. Mêmes apports ả l'origine, même renouvellement en 1966 que dans le traitement précédent. On a, en plus, apporté une fertilisation azotée à base d'ammonitrate au pied de chaque plant : $4 \mathrm{~g}$ d'azote en 1961, $4 \mathrm{~g}$ en 1962, $6 \mathrm{~g}$ en 1963 , $3,5 \mathrm{~g}$ en $1964,3,5 \mathrm{~g}$ en $1965,3,5 \mathrm{~g}$ en 1966.

- traitement $n^{\circ} 4:$ NPKCa profondeur. Les doses d'engrais appliquées sont les mêmes que dans le traitement 3 mais la dose initiale de phosphate a été appliquée pour moitié dans les $10 \mathrm{~cm}$ supérieurs et pour moitié à $40 \mathrm{~cm}$ de profondeur. (Jura).

La plantation a été effectuée au printemps 1961 avec des plants $2+2$ d'origine Massacre 


\author{
TABLEAU 1 \\ Essai du Lac de Guéry - Ponsses annuellex en hauteur (cm)
}

TABLE 1

Guéry Lake experinemt - Annwal height increements (cm), calculated F values, L.S.D., and effects of PKCa and $\mathrm{N}$

\begin{tabular}{|c|c|c|}
\hline $\begin{array}{l}\text { F Traitements théoriques } \\
\text { (theoretical F values) }\end{array}$ & $\begin{array}{l}\text { : à } 5 \% \\
\text { à } 11 \% \\
\text { à } 1 \% \%\end{array}$ & $\begin{array}{r}3,86 \\
6,55 \\
13,90\end{array}$ \\
\hline $\begin{array}{l}\text { Effets significatifs } \\
\text { (Significant effects) }\end{array}$ & $\begin{aligned}: \text { a } & 5 \% \\
\text { à } & 1 \% \\
\text { à } 1 \% & \%\end{aligned}$ & $\begin{array}{r}x \\
x x \\
x x x\end{array}$ \\
\hline
\end{tabular}

\begin{tabular}{|c|c|c|c|c|c|c|c|c|c|c|c|}
\hline \multirow{2}{*}{ Année } & \multirow{2}{*}{$\begin{array}{l}\text { Age } \\
\text { (1) }\end{array}$} & \multicolumn{4}{|c|}{ Traitements } & \multirow{2}{*}{$\begin{array}{c}\text { F } \\
\text { calculé }\end{array}$} & \multicolumn{3}{|c|}{ ppds (L.S.D.) } & \multicolumn{2}{|c|}{ Effet de } \\
\hline & & $\begin{array}{c}1 \\
\text { témoin }\end{array}$ & $\mathrm{PKCa}^{2}$ & $\underset{\text { (su) }}{\mathrm{NPKCa}^{3}}$ & $\underset{\text { (p) }}{\mathrm{NPKCa}^{4}}$ & & $5 \%$ & $1 \%$ & $1 \%$ & $\mathrm{PKCa}$ & $\mathrm{N}$ \\
\hline \multicolumn{12}{|l|}{ Pousse } \\
\hline 1962 & 2 & 2.46 & 4,17 & 5,84 & 6,23 & 44,49 & 1,22 & 1.75 & 2,58 & $\mathrm{x}$ & $\mathrm{x}$ \\
\hline 1963 & 3 & 4,37 & 10,68 & 12,51 & 12,16 & 23,92 & 2,49 & 3,57 & 5,26 & $\mathrm{xxx}$ & \\
\hline 1964 & 4 & 4,59 & 8.81 & 10.50 & 9,89 & 44,40 & 1,87 & 2,69 & 3,96 & $\mathrm{xxx}$ & \\
\hline 1965 & 5 & 5,39 & 9.26 & 11,12 & 10,84 & 34,03 & 1,44 & 2,08 & 3,05 & $x x x$ & $\mathbf{x}$ \\
\hline 1966 & 6 & 8,40 & 11,07 & 13,80 & 12,15 & 13,79 & 1,94 & 2,79 & - & $\mathrm{x}$ & $\mathrm{x}$ \\
\hline 1967 & 7 & 11,62 & 14,29 & 15,35 & 14,74 & 26,03 & 1,03 & 1,48 & 2,18 & $\mathbf{x x x}$ & $\mathrm{x}$ \\
\hline 1968 & 8 & 13,84 & 17,15 & 17,14 & 16,82 & 27,29 & 0,98 & 1,41 & 2,07 & $x \times x$ & \\
\hline 1969 & 9 & 15,70 & 19,04 & 19,30 & 19,83 & 10,52 & 1,84 & 2,65 & - & $\mathbf{x x}$ & \\
\hline 1970 & 10 & 22,47 & 28,11 & 28,10 & 29,69 & 19,74 & 2,28 & 3,28 & 4,81 & $\mathrm{xxx}$ & \\
\hline $\begin{array}{l}\text { Haut. tot. } \\
1970\end{array}$ & 10 & 111,41 & 145,83 & 154,79 & 149.51 & 19,44 & 14,19 & 20,41 & 30,02 & $\mathrm{xxx}$ & \\
\hline
\end{tabular}

(1) depuis plantation (after planting)

\title{
b) Résultats
}

Ils sont consignés dans le tableau 1, l'interprétation étant faite sur la base des moyennes des placeaux unitaires; ils confirment les conclusions de 1965.

L'effet de la fertilisation reste net, significatif a $1 \%$ la plupart du temps, sur la pousse annuelle et sur la hauteur totale. Il est dû pour l'essentiel aux trois éléments P, K, Ca sans qu'on puisse dissocier l'effet propre de chacun. L'effet de l'azote est faible et inconstant : pendant la période où ont été effectués des apports d'ammonitrate, il n'est significatif que 3 années sur 5; après le dernier épandage de 1966, il persiste faiblement pendant un an, puis disparaît; son effet global sur la hauteur totale n'est pas significatif après 9 ans.

Les analyses foliaires montrent que la nutrition des témoins en azote est très fluctuante; tantôt très mauvaise, surtout dans les premières années et en 1970, tantôt satisfaisante comme en 1964. La seule application des éléments P, K et Ca améliore très notablement la nutrition azotée, probablement par accélération de la minéralisation de la matière organique : ceci explique le faible effet des engrais azotés appliqués en complément de $\mathrm{P}, \mathrm{K}$ et $\mathrm{Ca}$, malgré une amélioration légère de la teneur en azote des aiguilles dans les traitements NPKCa tant que durent les applications d'ammonitrate (analyses de 1962, 63, 64). 
TABLEAU 2

Essai du Lac de Guéry - Analyses foliaires

(en $\%$ de la matière sèche)

TABLE 2

Guéry Lake Experiment - Nurrients concentrations in one year needlex (\% of D.W.)

\begin{tabular}{|c|c|c|c|c|c|c|c|c|}
\hline Eléments & $\begin{array}{l}\text { Année } \\
\text { Age (1) }\end{array}$ & $\begin{array}{c}1962 \\
2\end{array}$ & $\begin{array}{c}1963 \\
3\end{array}$ & $\begin{array}{c}1964 \\
4\end{array}$ & $\begin{array}{c}1965 \\
5\end{array}$ & $\begin{array}{c}1968 \\
8\end{array}$ & $\begin{array}{c}1969 \\
9\end{array}$ & $\begin{array}{c}1970 \\
10\end{array}$ \\
\hline $\mathrm{N}$ & $\begin{array}{c}\text { Traitements } \\
\text { témoin } \\
\text { PKCa } \\
\text { NPKCa }\end{array}$ & $\begin{array}{l}1,34 \\
1,82 \\
1,90\end{array}$ & $\begin{array}{l}1,21 \\
1,50 \\
2,03\end{array}$ & $\begin{array}{l}1,72 \\
1,42 \\
1,60\end{array}$ & $\begin{array}{l}1,41 \\
1,51 \\
-\end{array}$ & $\frac{1,50}{-}$ & $\frac{1,56}{1,50}$ & $\begin{array}{l}1,30 \\
1,57 \\
1,57\end{array}$ \\
\hline $\mathrm{P}_{2} \mathrm{O}_{4}$ & $\begin{array}{l}\text { tẻmoin } \\
\text { PKCa } \\
\text { NPKCa }\end{array}$ & $\begin{array}{l}0,29 \\
0,60 \\
0,55\end{array}$ & $\begin{array}{l}0.25 \\
0.40 \\
0.38\end{array}$ & $\begin{array}{l}0,31 \\
0,43 \\
0,39\end{array}$ & $\begin{array}{l}0,40 \\
0,44 \\
-\end{array}$ & $\frac{0,46}{-}$ & $\frac{0,53}{0,73}$ & $\begin{array}{l}0,50 \\
0,62 \\
0,60\end{array}$ \\
\hline K & $\begin{array}{l}\text { témoin } \\
\text { PKCa } \\
\text { NPKCa }\end{array}$ & $\begin{array}{l}0,83 \\
0,89 \\
0,70\end{array}$ & $\begin{array}{l}0,69 \\
0,71 \\
0,68\end{array}$ & $\begin{array}{l}0,62 \\
0,70 \\
0,61\end{array}$ & $\begin{array}{l}0,72 \\
0,72 \\
-\end{array}$ & $\frac{0,75}{-}$ & $\begin{array}{l}0,73 \\
0,76\end{array}$ & $\begin{array}{l}0,66 \\
0.69 \\
0,65\end{array}$ \\
\hline
\end{tabular}

(1) depuis plantation

La nutrition en phosphore, très déficiente dans les témoins au cours des premières années, s'est nettement améliorée à partir de 1965, ce qui n'empêche pas le nouvel apport de phosphates de 1966 d'avoir encore augmenté la teneur en $\mathrm{P}_{2} \mathrm{O}_{5}$ des arbres traités (analyses de 1969). Ceci corrobore assez bien l'effet des engrais sur la pousse des dernières années : alors que le supplément de croissance dú à $\mathrm{PK}$ et $\mathrm{Ca}$ atteignait 70 à $100 \%$ pendant les quatre premières années, il se limite à $25 \%$ environ depuis 1969.

La nutrition en $\mathrm{K}$ est toujours très suffisante.

\section{2. - Essai du contrat De Chalus}

Mis en place au début de 1963, sur une plantation de 1961, il se situe en Lozère, sur le granite porphyroïde de Margeride, dans une lande à callune et myrtille très pauvre, à $1320 \mathrm{~m}$ d'altitude. Le sol, malgré la différence de roche-mẻre, paraît voisin de celui du Lac de Guéry : sol cryptopodzolique très humifère et très désaturé, à teneur moyenne en potassium et assez bien pourvu apparemment en $\mathrm{P}_{2} \mathrm{O}_{5}(0,35$ à $0,50 \%$ \% $)$.

Trois traitements seulement ont été appliqués, avec 10 répétitions :

- traitement no $I$ : témoin,

- traitement $n^{\circ} 2$ : application d'un engrais complet soluble au pied de chaque plant, au printemps des années 1963, 1964 et 1965, apportant au total, pour ces 3 années et par plant, $15 \mathrm{~g}$ de $\mathrm{N}, 41 \mathrm{~g}$ de $\mathrm{P}_{2} \mathrm{O}_{5}, 27 \mathrm{~g}$ de $\mathrm{K}_{2} \mathrm{O}, 24 \mathrm{~g}$ de $\mathrm{CaO}$,

- traitement $n^{\circ} 3$ : apport d'ammonitrate à 20,5\% seulement, aux mêmes dates, apportant au total $16 \mathrm{~g}$ de $\mathrm{N}$ et $15 \mathrm{~g}$ de $\mathrm{CaO}$ par plant. 


\section{TABLEAU 3 \\ Essai du contrat De Chalus - Pousses annuelles (cm) \\ TABLE 3}

De Chalus plantarion Experiment - Annual height increement $(\mathrm{cm})$, calculated F values and L.S.D.

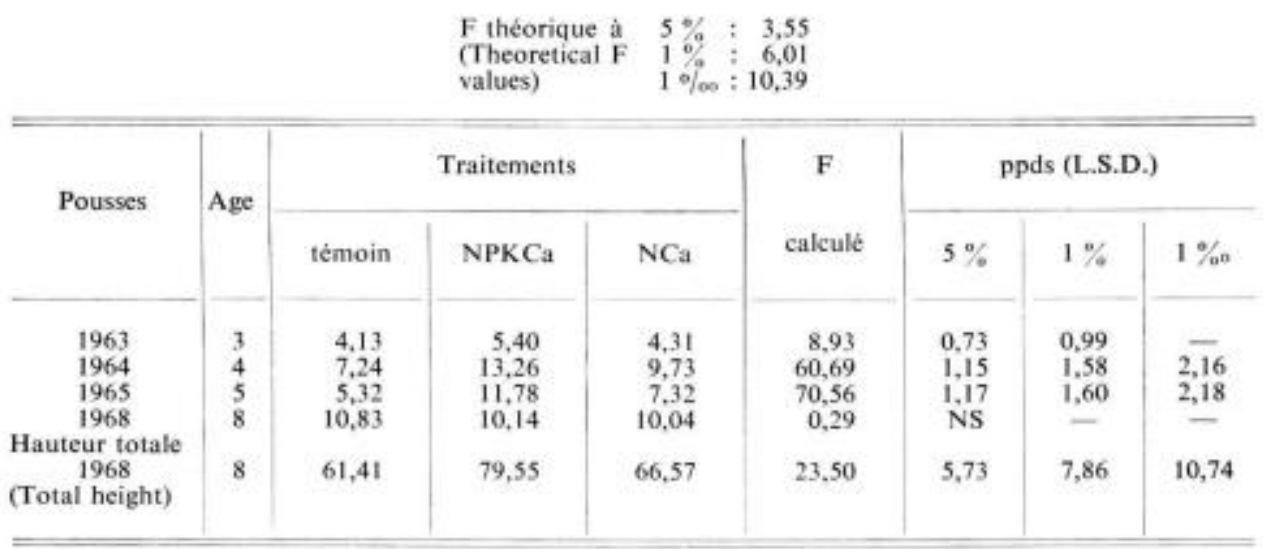

Les résultats, dont les premiers ont été publiés en 1965, sont indiqués au tableau 3. L'application d'azote et de calcium a déterminé pendant les 3 premières années une augmentation de croissance de l'ordre de $35 \%$ tandis qu'une application complémentaire de $\mathbf{P}$ et $\mathrm{K}$ et d'une dose plus forte de Ca entrainait un gain beaucoup plus considérable $(80 \%$ en $1964,120 \%$ en 1965).

TABLEAU 4

Contrat De Chalus - Analyses foliaires $(\%$ de la matière sèche)

TABLE 4

De Chalus plantation Experinent - Natrients concentrations in one year needles $(\%$ of D.W.)

\begin{tabular}{|c|c|c|c|c|}
\hline Élément & $\begin{array}{l}\text { Année } \\
\text { Age (1) }\end{array}$ & $\begin{array}{c}1964 \\
4\end{array}$ & $\begin{array}{c}1967 \\
7\end{array}$ & $\begin{array}{c}1970 \\
10\end{array}$ \\
\hline $\mathrm{N}$ & $\begin{array}{l}\text { Traitement } \\
\text { T } \\
\mathrm{NCa} \\
\mathrm{NPKCa}\end{array}$ & $\begin{array}{l}0,63 \\
2,07 \\
2,20\end{array}$ & 1.16 & $\frac{1,32}{-}$ \\
\hline $\mathrm{P}_{2} \mathrm{O}_{\mathrm{B}}$ & $\begin{array}{l}\text { T } \\
\text { NCa } \\
\text { NPKCa }\end{array}$ & $\begin{array}{l}0,34 \\
0,35 \\
0,49\end{array}$ & 0.36 & $\underline{0.47}$ \\
\hline K & $\begin{array}{l}\mathrm{T} \\
\mathrm{NCa} \\
\mathrm{NPKCa}\end{array}$ & $\begin{array}{l}0,79 \\
0,46 \\
0,80\end{array}$ & 0,54 & $\frac{0,68}{-}$ \\
\hline
\end{tabular}

(1) Depuis la plantation (after planting) 
Malheureusement, cet effet n'est que très temporaire : on ne constate plus aucune action de la fertilisation sur la pousse de 1968; seul subsiste un effet important sur la hauteur totale, qui ne représente donc que la conservation du gain acquis au cours des premières années. Les plants traités qui avaient nettement reverdi après l'application des engrais, ont d'ailleurs repris la couleur jaune caractéristique de la carence en azote. La pousse annuelle est d'ailleurs très faible ( $10 \mathrm{~cm}$ environ).

Les analyses foliaires (tableau 4) reflètent parfaitement cette extrême carence en azote. En 1964, la teneur en azote des aiguilles des témoins était extrêmement basse; en 1970, bien qu'améliorée, elle reste nettement inférieure à la normale. La nutrition en phosphore, assez faible au début et améliorée par le traitement NPKCa, s'est d'elle-même rétablie par la suite à un niveau normal. La teneur en K est toujours assez satisfaisante malgré une légère baisse par dilution, en 1964, dans le traitement NCa.

\section{3. - Essai d'Arzenc-de-Randon}

\section{a) Protocole}

Cet essai a été mis en place dans un terrain domanial tout proche du contrat De Chalus et sur un sol identique; sur les 5 répétitions, deux sont cependant nettement plus humides. Le sol a été décapé et sous-solé et les engrais, sauf les engrais azotés, ont été appliqués sur le fond des raies de décapage et légèrement enfouis par un binage manuel à la fin de l'été précédant la plantation (printemps 1964). Le but de cette expérience était d'obtenir des conclusions sur l'effet de chacun des éléments $\mathrm{N}, \mathrm{P}, \mathrm{K}$ et Ca qui $\mathrm{n}$ 'avait pas pu être isolé dans les essais précédents. Un dispositif factoriel $2^{4}$ à 16 traitements demandant trop de terrain, nous nous sommes contentés d'appliquer les 10 traitements suivants :

1 - témoin,

2 - $\mathrm{PCa}_{1}: 60 \mathrm{~g}$ de phosphate naturel broyé par mètre de bande décapée, soit environ $30 \mathrm{~g}$ de $\mathrm{P}_{2} \mathrm{O}_{5}$ et $45 \mathrm{~g}$ de $\mathrm{CaO}$ par $\mathrm{m}^{2}$ et $75 \mathrm{~kg}$ de $\mathrm{P}_{2} \mathrm{O}_{5}$ et $112 \mathrm{~kg}$ de $\mathrm{CaO}$ par hectare de plantation,

3 - $\mathrm{N}$ : simple apport de 10 à $15 \mathrm{~g}$ d'ammonitrate à $34,5 \%$ au pied de chaque plant en surface au printemps des années 1965, 1966, 1967, 1968 soit au total, pour ces quatre années, $15 \mathrm{~g}$ de $\mathrm{N}$ par plant,

$4-\mathrm{Ca}_{2}: 80 \mathrm{~g}$ de chaux éteinte par mètre linéaire de bande, soit $90 \mathrm{~g}$ environ de $\mathrm{CaO}$ par $\mathrm{m}^{2}$ et $225 \mathrm{~kg}$ par hectare de plantation. Cette dose initiale a été complétée en surface, en 1967, par un nouvel apport de $125 \mathrm{~g}$ de chaux par mètre de bande $\left(130 \mathrm{~g}\right.$ de $\mathrm{CaO}$ par $\mathrm{m}^{2}$ ou $325 \mathrm{~kg}$ par ha de plantation). Au total, ces deux doses correspondent à $11 \mathrm{~m} . e$. de Ca pour $100 \mathrm{~g}$ de sol qui saturent le complexe absorbant à $60 \%$ environ si l'on estime la profondeur chaulée à $10 \mathrm{~cm}$,

5 - K : Apport de $30 \mathrm{~g}$ de chlorure de potassium par mètre linéaire $\left(30 \mathrm{~g}\right.$ de $\mathrm{K}_{2} \mathrm{O}$ par $\mathrm{m}^{2}$ ou $75 \mathrm{~kg}$ par ha de plantation),

$6-\mathrm{Ca}_{2}+\mathrm{N}$ : association des traitements $\mathrm{Ca}_{2}$ et $\mathrm{N}$,

$7-\mathrm{K}+\mathrm{N}$,

$8-\mathrm{Ca}_{2}+\mathrm{K}+\mathrm{N}$. 
NUTRITION DE L'ÉPICÉA DANS LE MASSIF CENTRAL

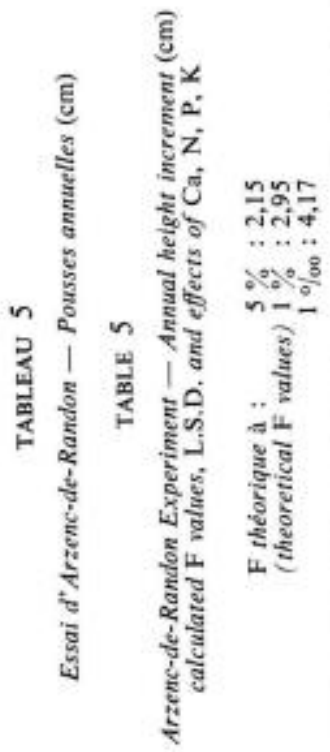

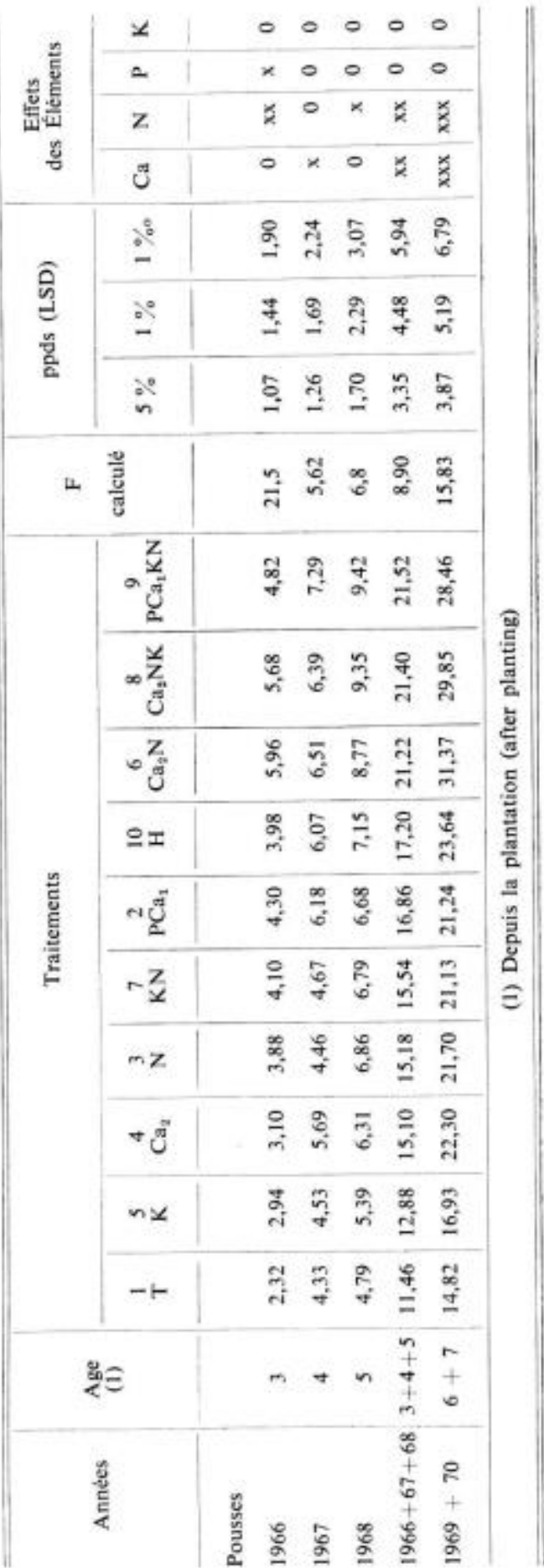




$$
9-\mathrm{PCa}_{1}+\mathrm{K}+\mathrm{N} \text {, }
$$

$10-\mathrm{H}$ : apport dans le trou de plantation, au moment de la mise en place du plant, de $1 \mathrm{dm}^{3}$ environ d'un mor de pessière artificielle, prélevé à quelques kilomètres de l'essai à la même altitude et dans des conditions écologiques comparables; on espérait ainsi déclencher ou favoriser la mycorhization des plants; les quantités d'éléments nutritifs apportés par cet humus étaient faibles, de l'ordre de $1 \mathrm{~g}$ de $\mathrm{N}, 0,2$ à $0,3 \mathrm{~g}$ de $\mathrm{P}_{2} \mathrm{O}_{5}, \mathrm{~K}_{2} \mathrm{O}$ et $\mathrm{CaO}$.

Les plants étaient d'origine Massacre (Jura).

TABLEAU 6

Essais d'Arzenc-de-Randon - Analyses foliaires (en $\%$ de la matiere seche)

TABLE 6

Arzenc-de-Randon Experiment - Nutrients concentrations in one year needles $(\%$ of D.W.

\begin{tabular}{|c|c|c|c|c|c|}
\hline Êléments & $\begin{array}{l}\text { Année } \\
\text { Age (1) }\end{array}$ & $\begin{array}{c}1967 \\
4\end{array}$ & $\begin{array}{c}1968 \\
5\end{array}$ & $\begin{array}{c}1969 \\
6\end{array}$ & $\begin{array}{c}1970 \\
7\end{array}$ \\
\hline $\mathrm{N}$ & $\begin{array}{l}\mathrm{T} \\
\mathrm{Ca} \\
\mathrm{PCa} \\
\mathrm{CaN} \\
\mathrm{PCaKN} \\
\mathrm{H}\end{array}$ & $\begin{array}{l}1,27 \\
1,31 \\
1,31 \\
-\end{array}$ & $\begin{array}{l}1,09 \\
1,18 \\
1,10 \\
1,23 \\
1,11\end{array}$ & $\frac{1,09}{\overline{1,06}} \frac{}{1,01}$ & $\begin{array}{l}1,06 \\
1,06 \\
-\overline{0,98} \\
0,95 \\
1,03\end{array}$ \\
\hline $\mathrm{P}_{2} \mathrm{O}_{2}$ & $\begin{array}{l}\mathrm{T} \\
\mathrm{Ca} \\
\mathrm{PCa} \\
\mathrm{CaN} \\
\mathrm{PCaKN} \\
\mathrm{H}\end{array}$ & $\begin{array}{c}0,39 \\
0,34 \\
0,38 \\
-\end{array}$ & $\begin{array}{l}0,42 \\
0,45 \\
0,42 \\
0,38 \\
-\overline{0,38}\end{array}$ & $\begin{array}{l}\frac{0,52}{-} \\
\frac{0,50}{\overline{0}} \\
0,50\end{array}$ & $\begin{array}{l}0,44 \\
0,54 \\
0,47 \\
0,63 \\
0,43\end{array}$ \\
\hline $\mathbf{K}$ & $\begin{array}{l}\mathrm{T} \\
\mathrm{Ca} \\
\mathrm{PCa} \\
\mathrm{CaN} \\
\mathrm{PCaKN} \\
\mathrm{H}\end{array}$ & $\begin{array}{l}0,63 \\
0,62 \\
0,68 \\
\end{array}$ & $\begin{array}{l}0,66 \\
0,59 \\
0,68 \\
0,60 \\
\overline{0,70}\end{array}$ & $\begin{array}{l}\frac{0,71}{\overline{-}} \\
\overline{0,69} \\
0,74\end{array}$ & $\begin{array}{l}0,58 \\
0,65 \\
- \\
0,47 \\
0,66 \\
0,65\end{array}$ \\
\hline $\mathrm{Ca}$ & $\begin{array}{l}\mathrm{T} \\
\mathrm{Ca} \\
\mathrm{PCa} \\
\mathrm{CaN} \\
\mathrm{PCaKN}_{\mathrm{H}} \\
\mathrm{H}\end{array}$ & $\begin{array}{c}0,41 \\
0,61 \\
0,37 \\
-\end{array}$ & $\begin{array}{l}0,45 \\
0,48 \\
0,61 \\
0,52 \\
\overline{0,48}\end{array}$ & $\begin{array}{l}0,59 \\
\overline{-} \\
\overline{0,67} \\
\overline{0,67}\end{array}$ & $\begin{array}{l}0,59 \\
0,60 \\
\overline{0,64} \\
0,64 \\
0,70\end{array}$ \\
\hline
\end{tabular}

(1) depuis la plantation (after planting)

\section{b) Résultats}

Les pousses annuelles sont dans l'ensemble très modestes, ce qui s'explique, en partie, 
par la brièveté de la saison de végétation. Comme le montre le tableau 5 , la fertilisation s'avère très efficace puisque l'effet des traitements est toujours significatif à $1 \%$.

- Effet du potassium : parmi les éléments testés, le potassium n'a aucun effet : le traitement $\mathrm{K}$ n'est jamais significatif par rapport au témoin, ni NK par rapport à $\mathrm{N}$, ni CaNK par rapport à $\mathrm{CaN}$.

- Effet du phosphore : le phosphore a un faible effet significatif sur la pousse en hauteur dans les premières années puisque, en $1966, \mathrm{PCa}_{1}$ diffère à $5 \% \mathrm{de} \mathrm{Ca}_{2} ;$ mais $\mathrm{PCa}_{1} \mathrm{KN}$ ne diffère pas de $\mathrm{Ca}_{2} \mathrm{KN}$. A partir de 1967 , on ne note plus de différence entre $\mathrm{PCa}_{1}$ et $\mathrm{Ca}_{2}$, mais $\mathrm{PCa}_{1}$ est plus significatif que $\mathrm{Ca}_{2}$ par rapport au témoin jusqu'en 1968 : un léger effet du phosphore reste done vraisemblable.

A partir de 1969 , la situation s'inverse et $\mathrm{Ca}_{2}$ devient supérieur à $\mathrm{PCa}_{1}$, bien que de manière non significative et sans que le degré de différence de $\mathrm{PCa}_{1}$ par rapport au témoin diminue. Il faut voir dans cette inversion l'effet renforcé du calcium dans le traitement $\mathrm{Ca}_{2}$ après les épandages complémentaires de 1967.

Les analyses foliaires montrent qu'après une insuffisance de nutrition en phosphore des témoins en 1967, la situation s'est améliorée; l'apport de phosphore ne relève d'ailleurs pas cette nutrition, sauf semble-t-il en présence d'azote et de potassium (analyses 1970).

- Effet du calcium : jusqu'en 1968, le calcium employé seul agit faiblement; le traitement Ca n'est significatif par rapport au témoin qu'en 1967. Par contre, il se révèle significatif à $1 \%$ sur la somme des pousses $1969+1970$ et l'on peut voir là l'effet de l'épandage complémentaire réalisé en 1967. Cet épandage avait été décidé à la suite de travaux de laboratoire qui montraient que la minéralisation de l'azote dans les sols de ce type ne pouvait être accélérée que par une addition importante de chaux (BONNEAU, 1967). Le regain d'effet du traitement $\mathrm{Ca}_{2}$ ne peut cependant être considéré que comme une confirmation partielle de ce point de vue car l'analyse foliaire n'en apporte pas la preuve directe, la nutrition en azote dans le traitement $\mathrm{Ca}_{2}$ restant à peu de chose près aussi catégoriquement mauvaise que dans le témoin.

- Effet de l'azote : l'azote, employé seul, a également un effet assez faible pendant les 3 premières années et en même temps très variable : significatif a $1 \%$ en 1966, à $5 \%$ en 1968, non significatif en 1967. Par contre, son action s'affirme en 1969 et 1970 où l'effet est significatif à $1 \%$. Rappelons que les apports d'engrais azotés ont été pratiqués pour la dernière fois en 1968: on est doncen présence d'un fort arrière-effet de l'azote. L'enseignement de l'essai voisin du contrat De Chalus, oủ l'effet de l'azote sur la pousse est devenu nul 3 ans après le dernier épandage de 1965 , nous incite, cependant, à penser que l'action de l'azote va rapidement disparaitre à Arzenc-de-Randon.

De tous les traitements expérimentés, $\mathrm{Ca}_{2} \mathrm{~N}, \mathrm{Ca}_{2} \mathrm{KN}$ et $\mathrm{PCa}_{1} \mathrm{KN}$ apparaissent comme les plus efficaces, différant de manière très significative des traitements $\mathrm{Ca}_{2}$ et $\mathrm{PCa}_{1}$, mais ils ne diffèrent pas les uns des autres (sauf en 1966), leur action devant par conséquent être attribuće au couple CaN. La croissance par rapport au témoin est doublée et l'effet global de CaN est sensiblement la somme des effets de $\mathrm{Ca}$ et de $\mathrm{N}$ sans qu'il $\mathrm{y}$ ait interaction significative entre ces deux éléments. Les analyses foliaires montrent que ce traitement très efficace $\mathrm{Ca}_{2} \mathrm{~N}$ ne relève cependant pas de manière sensible la nutrition azotée; le léger effet constatẻ en 1968 disparaît rapidement.

- Effet du traitement $H$ : ce traitement se révèle parfaitement efficace : son effet, 
significatif à $1 \%$ sur la somme des pousses $1966+1967+1968$ augmente ensuite et devient significatif à $1 \%$, permettant un gain de croissance en hauteur du même ordre de grandeur que le traitement $\mathrm{Ca}$. L'examen de quelques racines a montré que les témoins eux-mêmes étaient fortement mycorhizés, avec abondante ramification des racines et manteau de type $F$, tandis que les plants du traitement $\mathrm{H}$ avaient des racines moins mycorhizées et moins ramifiées avec des manteaux de type $\mathrm{B}^{1}$. L'effet du traitement $\mathrm{H}$ n'est donc pas facilement explicable et trois hypothèses peuvent être envisagées :

- les mycorhizes constatées sur les plants du traitement $\mathrm{H}$ sont la conséquence de l'apport d'humus de pessière et sont plus efficaces, malgré un développement morphologique moindre, que celles du témoin apportées de la pépinière ou constituées sur place avec un champignon présent dans le sol de la lande ;

- l'incorporation de l'humus lors de la plantation a conduit à une mise en place plus soigneuse des plants ;

- l'apport d'humus, presque négligeable sur le plan des éléments nutritifs, aurait, cependant, constitué un apport de matière hydrocarbonée susceptible de favoriser la fixation

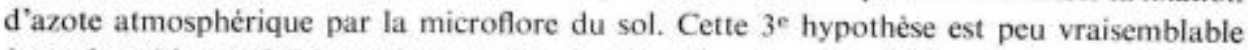
étant donnẻ le très faible rendement de la fixation d'azote atmosphérique et le type d'humus peu actif de ces sols d'altitude.

Quoiqu'il en soit, les analyses foliaires ne révèlent pas non plus, dans le cas de ce traitement, d'amélioration sensible de la nutrition.

Les résultats précédents ont été obtenus, nous l'avons dit, sur des plants d'origine Massacre (Jura). Mais les lignes de bordure de chaque parcelle unitaire ont été plantées d'épicéas provenant d'une pépinière locale, d'origine inconnue, et qui, dès le début, se sont montrés beaucoup plus vigoureux que les épicéas du Jura qui ont vraisemblablement souffert du transport et de la mise en jauge. Nous avons donc, par prudence, mesuré ces lignes de bordure : le classement des traitements reste le même mais leur effet par rapport au témoin est moindre : $15 \%$ de croissance supplémentaire pour le calcium seul et $50 \%$ pour le traitement $\mathrm{Ca}_{2} \mathrm{~N}$.

\section{c) Conclusions}

L'essentiel des enscignements de cet essai se résume ainsi : la fertilisation la plus efficace est une fertilisation $\mathrm{Ca}+\mathrm{N}$ permettant un gain de croissance en hauteur de $50 \%$ ou plus. Mais ce traitement suppose un apport copieux de calcium saturant le complexe à $50 \%$ au moins et une fertilisation azotée apportée régulièrement pendant plusieurs années.

Des traitements plus simples, tels que le chaulage seul ou l'apport d'un humus de pessière, apportent une amélioration sensible, bien que moindre, de la croissance. En revanche, ils ne nécessitent pas, comme les apports d'engrais azotés, d'intervention fréquente. Quel que soit le traitement appliqué, la nutrition azotée reste désespérément mauvaise.

1. Analyses effectuées par Mme RouqueroL, C.R.A.S.E. Montpellier, que nous remercions vivement de son aide et de l'intérét qu'elle a manifesté pour le sujet. 


\section{4. - Essai des Ygrands}

\section{a) Situation}

Il est situé dans le Cantal, au Sud de Chaudesaigues (Commune de Jabrun), à $1100 \mathrm{~m}$ d'altitude, à la pointe Nord-Ouest de la Margeride, sur un granite porphyroïde très quartzeux, dans une parcelle dépendant d'une ancienne ferme, autrefois pâturée et probablement cultivée, et retournée à la lande à callune.

Le sol est du type brun acide, avec un horizon $\mathrm{A}_{1}$ peu épais ( $10 \mathrm{~cm}$ environ), mais riche en matière organique $(25 \%)$, avec un $\mathrm{C} / \mathrm{N}$ qui peut varier de 20 à 40 suivant les endroits. C'est un sol assez bien pourvu en calcium échangeable du fait sans doute de l'ancienne culture (4 à 6 m.e. pour $100 \mathrm{~g}$ en $\mathrm{A}_{1}, 0,6$ à 1,2 m.e. en (B)), moyennement riche en potassium ( 1 m.e. en $A_{1}$ et 0,15 en (B)), mais plutôt pauvre en phosphore, surtout si on le compare aux sols d'Arzenc-de-Randon et du Lac de Guéry $\left(0,06\right.$ à $0,08 \%$ de $\left.\mathrm{P}_{2} \mathrm{O}_{5}\right)$.

\section{b) Dispositif}

Une plantation d'Épicéa a été effectuée par les soins de la SOMIVAL après passage de rotavator et sous-solage en automne 1965.

En 1967, donc au cours de la $2^{\circ}$ année de végétation, on a mis en place un dispositif expérimental du type factoriel $2^{4}$ avec les éléments $\mathrm{N}, \mathrm{P}, \mathrm{K}$ et Ca représentés au niveau zéro et à un niveau d'apport caractérisé comme suit :

$\mathrm{N}: 10 \mathrm{~g}$ d'ammonitrate à $34,5 \%$ apportés en surface autour de chaque plant, au printemps des années 1968, 1969 et 1970;

P : $40 \mathrm{~g}$ de superphosphate triple par plant, épandus en surface, à l'automne 1967, sur $0,25 \mathrm{~m}^{2}$ environ;

$\mathrm{K}: 20 \mathrm{~g}$ de sulfate de potassium, appliqués comme le superphosphate;

$\mathrm{Ca}: 60 \mathrm{~g}$ de chaux éteinte appliqués de la mème façon.

Chacun des 16 traitements a été répété 7 fois sur de petites parcelles contenant chacune 36 plants mesurables.

\section{c) Résultats}

IIs sont indiqués aux tableaux 7 (pousses annuelles) et 8 (analyses foliaires). L'interprétation, effectuée sur la somme des pousses 1968 et 1969, montre un effet très important de la fertilisation. Les meilleurs traitements sont NPK, NPKCa et NP, qui ne diffèrent pas les uns des autres et assurent un gain de croissance en hauteur de $49 \%$ environ. Le traitement NPCa les suit de très près, si bien qu'au total, on trouve en tête tous les traitements associant $\mathrm{N}$ et $\mathrm{P}$. L'interprétation détaillée du dispositif factoriel montre que les effets de $\mathrm{N}$ et $\mathrm{P}$ sont de loin les plus importants, largement significatifs à $1 \%$, que l'interaction $\mathrm{N} \times \mathrm{P}$ est également forte (significative à $1 \%$ ), tandis que l'interaction $\mathrm{P} \times \mathrm{K}$ n'est significative qu'à $5 \%$. 


\section{TABLEAU 7}

Essai des Ygrands - pousses annuelles (cm)

TABLE 7

Les Ygrands Experiment : annual height increment $(\mathrm{cm})$, calculated $\mathrm{F}$ value

F calculé pour $1968+1969: 6,28$

F théorique à $5 \%: 1,79$ ppds $5 \%: 3,54$

(theoretical F values) $1 \%: 2,27$ (L.S.D.) $1 \%: 4,68$

\begin{tabular}{|c|c|c|c|c|c|c|c|}
\hline $\begin{array}{l}\text { Année } \\
\text { Age (1) }\end{array}$ & $\begin{array}{c}1968 \\
3\end{array}$ & $\begin{array}{l}1969 \\
r 4\end{array}$ & $\begin{aligned} 1968 & +1969 \\
3 & +4\end{aligned}$ & $\begin{array}{c}\text { Année } \\
\text { Age (1) }\end{array}$ & $\begin{array}{c}1968 \\
3\end{array}$ & $\begin{array}{c}1969 \\
4\end{array}$ & $\begin{array}{c}1968+1969 \\
3+4\end{array}$ \\
\hline $\begin{array}{l}\text { Traitement } \\
\text { T } \\
\mathrm{N} \\
\mathrm{P} \\
\mathrm{K} \\
\mathrm{Ca} \\
\mathrm{NP} \\
\mathrm{NK} \\
\mathrm{NCa}\end{array}$ & $\begin{array}{r}9,14 \\
9,25 \\
8,76 \\
9,71 \\
10,12 \\
11,58 \\
8,52 \\
10,23\end{array}$ & $\begin{array}{r}9,68 \\
11,91 \\
10,68 \\
9,39 \\
10,48 \\
15,35 \\
11,20 \\
13,28\end{array}$ & $\begin{array}{l}18,82 \\
21,16 \\
21,00 \\
19,10 \\
20,60 \\
26,93 \\
19,72 \\
23,51\end{array}$ & $\begin{array}{l}\text { Traitement } \\
\text { PK } \\
\text { PCa } \\
\text { KCa } \\
\text { NPK } \\
\text { NPCa } \\
\text { NKCa } \\
\text { PKCa } \\
\text { NPKCa }\end{array}$ & $\begin{array}{l}10,75 \\
11,08 \\
10,55 \\
12,28 \\
10,63 \\
10,06 \\
12,02 \\
11,65\end{array}$ & $\begin{array}{l}11,82 \\
10,58 \\
10,83 \\
17,28 \\
15,04 \\
11,62 \\
12,82 \\
16,10\end{array}$ & $\begin{array}{l}22,57 \\
21,66 \\
21,38 \\
29,56 \\
25,67 \\
21,68 \\
24,84 \\
27,75\end{array}$ \\
\hline
\end{tabular}

(1) Depuis la plantation (after planting)

\section{TABLEAU 8}

Essai des Ygrands - Analyses foliaires

(\% de la matière sèche), en 1970 ( 5 ans après la plantation)

TABLE 8

Les Ygrands Experiment - Nutrient concentrations in one year needles $\%$ of D.W.), in 1970 (5 years after planting)

\begin{tabular}{|c|c|c|c|c|}
\hline \multirow{2}{*}{ Traitements } & \multicolumn{4}{|c|}{ Élémen ts } \\
\hline & $\mathrm{N}$ & $\mathrm{P}_{2} \mathrm{O}_{\mathrm{b}}$ & K & $\mathrm{Ca}$ \\
\hline $\mathrm{T}$ & 0,90 & 0,28 & 0,65 & 0,70 \\
\hline $\mathrm{NCa}$ & 0,89 & 0,20 & 0,60 & 0,67 \\
\hline $\mathrm{NPCa}$ & 0,94 & 0,32 & 0,56 & 0,64 \\
\hline
\end{tabular}

L'analyse foliaire des témoins (tableau 8) fait parfaitement ressortir la carence en azote et en phosphore. Dans le traitement NPCa, le seul des traitements très actifs dont les aiguilles aient été analysées, la nutrition en phosphore et surtout en azote reste très insuffisante. De nouvelles interventions seront nécessaires pour l'amener à l'optimum. 


\section{5. - Conclusions partielles}

Les quatre essais décrits ci-dessus montrent que la fertilisation peut améliorer considérablement la croissance des jeunes plantations d'Épicéa dans les stations d'altitude; le gain de croissance annuelle en hauteur peut varier de 25 a $50 \%$ suivant les stations. Si I'on excepte les roches-mères volcaniques riches (basaltes), l'azote y joue le rôle essentiel mais son effet est fortement renforcé par un apport de calcium ou de phosphore suivant les cas. Malheureusement, il semble que l'effet de l'azote disparaisse quelques années après qu'on ait arrêté les épandages annuels; l'effet des seuls apports de calcium ou de phosphore n'assurerait qu'un gain beaucoup plus faible ( 20 à $25 \%$ environ). Celà pose un problème pratique, le coût d'épandages répétés d'engrais azotés étant hors de proportion avec le gain de croissance en valeur absolue qui reste forcément faible dans ces stations à courte saison de végétation. Bien plus, les épandages d'azote effectués s'avèrent incapables de surmonter l'énorme carence azotée qui sévit dans ces stations : ils ont été suffisants pour augmenter notablement la croissance, mais encore trop faibles pour assurer une nutrition normale. Il semble qu'il ne pourrait y avoir de solution que dans l'apport périodique de fortes doses d'engrais azotés lentement minéralisables.

La cause de cette carence azotée réside dans le faible taux de minéralisation annuel de la matière organique, dont le $\mathrm{C} / \mathrm{N}$ est cependant bas; cette faiblesse de la minéralisation reste elle-même inexpliquée. L'hypothèse d'une toxicité aluminique directe qui gênerait le développement racinaire et l'alimentation des épicéas a été rejetée lors d'études antérieures (BONNEAU, 1965).

Par ailleurs, un apport dans la terre de plantation d'humus forestier riche en mycélium a eu un effet à la fois durable et assez important. Il semble prouvé que la mycorhisation dans ce traitement est d'une nature différente de celle des témoins, sans être toutefois plus abondante. Un effet réel « mycorhisation », sans être certain, n'est donc pas exclu.

\section{II. - LES EXPÊRIMENTATIONS A MOYENNE ALTITUDE}

Nous disposons de 3 essais de terrain à moyenne altitude : Saint-Apollinaire (Rhône), Razès (Hte-Vienne) et Grand-Grammont (Hte-Vienne) et d'un essai en pots sur un sol de St-Sétiers (Corrèze).

\section{1. - Essai de Saint-Apollinaire}

Disons immédiatement, pour épargner du temps au lecteur pressé, que la fertilisation n'a aucun effet dans cet essai. Nous donnerons cependant les grandes lignes des conditions écologiques et des traitements.

L'expérience a été installée, en profitant d'une opération du Fonds Forestier National, dans une lande à fougère aigle et graminées, à $800 \mathrm{~m}$ d'altitude, sur un substratum granitique; une partie de cette lande a été anciennement cultivée. Le sol, du type brun acide, est assez riche en matière organique ( 7 à $9 \%$ en surface, 2 à $6 \%$ à $30 \mathrm{~cm}$ de profondeur), et l'arène granitique apparaît vers $40 \mathrm{~cm}$. La texture est sablo-limono-argileuse. La fertilité est bonne : 0,30 à $1 \%$ de $\mathrm{P}_{2} \mathrm{O}_{5}$ suivant les horizons, 0,2 à $0,8 \mathrm{~m}$.e de potassium échangeable pour $100 \mathrm{~g}$, 0,4 à 2 m.e de calcium. Le C/N est bas : 10 à 11 . 
On a appliqué 12 traitements qui sont la combinaison factorielle de 4 traitements non azotés et de 3 traitements azotés.

\section{- Traitements non azotés:}

T : témoin.

$\mathrm{P} \quad: 20 \mathrm{~g}$ de $\mathrm{P}_{2} \mathrm{O}_{5}$ par $\mathrm{m}^{2}$ épandus sur les raies de décapage, en automne 1962, avant plantation (phosphate de chaux), puis $8 \mathrm{~g}$ au pied de chaque plant en 1966 (superphosphate).

Ca : calcaire broyé : $110 \mathrm{~g}$ de $\mathrm{CaO}$ par $\mathrm{m}^{2}$, puis $53 \mathrm{~g}$ par plant comme précédemment. $\mathrm{CaK}$; au traitement Ca précédent, on a ajouté $30 \mathrm{~g}$ de $\mathrm{K}_{2} \mathrm{O}$ par $\mathrm{m}^{2}$ (sulfate de potassium), puis $7 \mathrm{~g}$ par plant en 1966 (Patentkali apportant en outre $2,5 \mathrm{~g} \mathrm{de} \mathrm{MgO}$ ).

\section{- Traitement azotés :}

$\mathrm{O} \quad$ : aucun apport.

$\mathrm{N}_{1}: 3,4 \mathrm{~g}$ d'azote en surface au pied de chaque plant, au printemps des années 1964 , 1965 et 1966, sous forme d'ammonitrate.

$\mathrm{N}_{2}$ : même apport sous forme de sulfate d'ammoniaque.

Aucun des 12 traitements appliqués n'a eu d'effet, la croissance annuelle s'avérant satisfaisante : $7 \mathrm{~cm}$ la première année, $14 \mathrm{~cm}$ la $2^{e}$ année, $20 \mathrm{~cm}$ la $3^{e}$ année, $29 \mathrm{~cm}$ la $4^{e}$ année et $35 \mathrm{~cm}$ la $5^{e}$ année. Comme nous l'avons dit, le sol pouvait a priori être considéré comme fertile et le manque d'effet de la fertilisation n'a donc rien de surprenant.

\section{2. - Essai de Razes}

\section{a) Conditions écologiques}

Il est installé sur un sol brun humifère, sur granite à 2 micas, dans une lande à callune très typique, sporadiquement mélangée de fougère. Ce sol comprend un horizon humifère de $20 \mathrm{~cm}$ d'épaisseur contenant $15 \%$ de matière organique en surface et dont le $\mathrm{C} / \mathrm{N}$ est de l'ordre de 25 , bien qu'il ne s'agisse pas d'un humus brut typique, l'horizon $\mathrm{A}_{0}$ développé sous la callune ayant été éliminé avec elle par le travail du sol. La texture est sablo-argileuse; la richesse en phosphore parait élevée $(0,25 \%$ en surface à $1 \%$ en profondeur) tandis que la teneur en potassium échangeable est plutôt faible $(0,15 \mathrm{~m}$.e pour $100 \mathrm{~g}, 0,07$ en profondeur) et le calcium presqu'absent $(0,05$ à $0,10 \mathrm{~m} . \mathrm{e})$. Le pH est cependant modérément acide ( 5 environ). La teneur en potassium est en fait assez. fluctuante et reflète sans doute l'intensité des exploitations anciennes de callune.

\section{b) Dispositif}

II s'agit d'un essai en blocs complets avec 8 répétitions et 7 traitements :

$$
\begin{array}{llll}
\text { T } & \mathrm{Ca} & \mathrm{CaP} & \mathrm{CaPK} \\
& \mathrm{CaN} & \mathrm{CaPN} & \mathrm{CaPKN}
\end{array}
$$

Ces 7 traitements sont les additions des applications élémentaires suivantes :

$\mathrm{Ca}: 35 \mathrm{~g}$ de $\mathrm{CaO}$ par plant, sous forme de chaux éteinte épandue en surface autour du plant (sur 30 à $40 \mathrm{~cm}$ de rayon), puis enfouic par un léger binage quelques semaines après la plantation en mars 1967;

P : $18 \mathrm{~g}$ de $\mathrm{P}_{2} \mathrm{O}_{5}$ sous forme de superphosphate triple à $48 \%$ appliqué comme Ca; 
NUTRITION DE L'ÉPICÉA DANS LE MASSIF CENTRAL

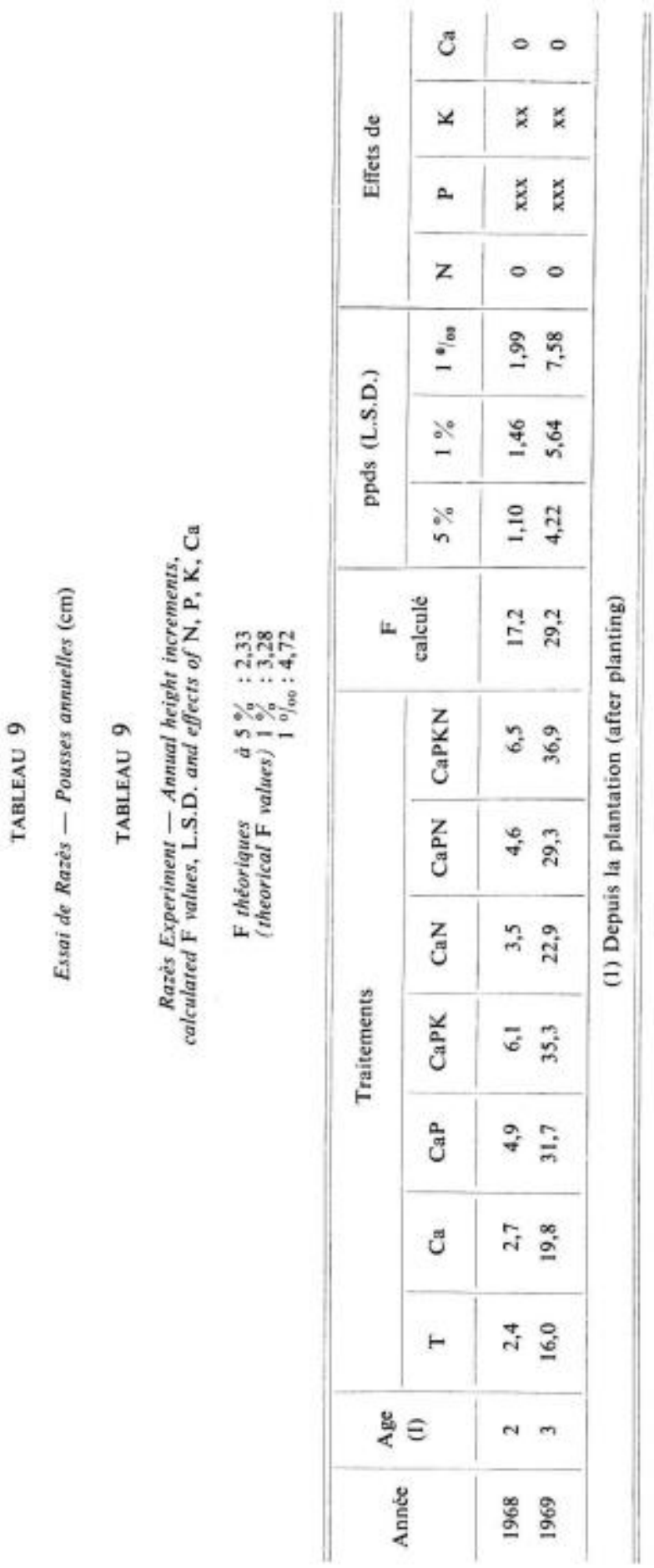


$\mathrm{K}: 10 \mathrm{~g}$ de $\mathrm{K}_{2} \mathrm{O}$ sous forme de sulfate de potasse appliquée de la même façon ;

$\mathrm{N}: 4,5 \mathrm{~g}$ d'azote au printemps 1968 (urée), 3,5 g au printemps 1969 (ammonitrate à $34,5 \%$ ),

$5 \mathrm{~g}$ au printemps 1970 (ammonitrate) épandus autour des plants et en surface.

\section{c) Résultats}

Le tableau 9 indique les pousses annuelles de 1968 à 1969. L'effet de la fertilisation est extrêmement net et très largement significatif à $1 \%$. Deux éléments apparaissent comme responsables de cette action : le phosphore et le potassium, la combinaison des deux (en addition avec le calcium) assurant un gain de croissance annuelle de plus de $100 \%$. Le phosphore a une action beaucoup plus marquée que celle du potassium, son effet moyen s'établissant en 1969 à $9,1 \mathrm{~cm}$ contre $5,6 \mathrm{~cm}$ pour le potassium.

TABLEAU 10

Essai de Razès - Analyses foliaires (en $\%$ de la matière séche)

TABLE 10

Razes Experiment - Nutrient concentrations in one year needles $(\%$ of D,W.)

\begin{tabular}{|c|c|c|c|}
\hline Éléments & $\begin{array}{l}\text { Annće } \\
\text { Age (1) }\end{array}$ & $\begin{array}{c}1968 \\
2\end{array}$ & $\begin{array}{c}1969 \\
3\end{array}$ \\
\hline $\mathrm{N}$ & $\begin{array}{l}\quad \text { Traitements } \\
\mathrm{T} \\
\mathrm{Ca} \\
\mathrm{CaP} \\
\mathrm{CaN} \\
\mathrm{CaPN} \\
\mathrm{CaPKN}\end{array}$ & 2,45 & $\begin{array}{l}1,66 \\
1,47 \\
\frac{1,89}{1,85}\end{array}$ \\
\hline $\mathrm{P}_{2} \mathrm{O}_{1}$ & $\begin{array}{l}\mathrm{T} \\
\mathrm{Ca} \\
\mathrm{CaP} \\
\mathrm{CaN} \\
\mathrm{CaPN} \\
\mathrm{CaPKN}\end{array}$ & 0,43 & $\begin{array}{l}0,27 \\
0,27 \\
\overline{0,29} \\
\overline{0,64}\end{array}$ \\
\hline K & $\begin{array}{l}\mathrm{T} \\
\mathrm{Ca} \\
\mathrm{CaP} \\
\mathrm{CaN} \\
\mathrm{CaPN} \\
\mathrm{CaPKN}\end{array}$ & 0,67 & $\begin{array}{l}0,61 \\
0,56 \\
\overline{0,44} \\
\overline{0,72}\end{array}$ \\
\hline $\mathrm{Ca}$ & $\begin{array}{l}\mathrm{T} \\
\mathrm{Ca} \\
\mathrm{CaP} \\
\mathrm{CaN} \\
\mathrm{CaPN} \\
\mathrm{CaPKN}\end{array}$ & 0,26 & $\begin{array}{l}0,14 \\
0,37 \\
\overline{0,32} \\
\overline{0,63}\end{array}$ \\
\hline
\end{tabular}

(1) Depuis la plantation (after planting) 
L'analyse foliaire des témoins (tableau 10) ne fait pas ressortir de carence évidente en 1968, tandis qu'en 1969 et 1970 la teneur des aiguilles en $\mathrm{P}_{2} \mathrm{O}_{5}$ apparaít comme faible. Cette teneur remonte considérablement pour les traitements qui comportent du phosphore. La nutrition en potassium des témoins semble a priori assez satisfaisante, mais l'effet de cet élément sur la croissance laisse prévoir que l'optimum est plus élevé que nous ne le supposions; les plants du traitement $\mathrm{CaPKN}$ ont une teneur nettement plus élevée que le témoin en 1969.

La nutrition azotée des témoins, surabondante en 1968, se dégrade en 1969; en 1969, l'apport d'engrais azoté la relève ce qui explique la tendance à I'amélioration de la croissance de CaN par rapport à Ca (positive mais non significative). La concentration en azote des aiguilles des témoins $(1,66 \%)$ doit ainsi être jugée comme légèrement inféricure à l'optimum.

Le calcium, bien que très peu abondant dans les aiguilles des témoins ne montre aucun effet significatif sur la croissance, malgré une absorption nettement plus forte dans les traitements où cet élément a été appliqué. II faut noter, cependant, que le traitement Ca est différent du témoin au seuil de $10 \%$ en 1969 et que, dans ces conditions, la teneur de $0,14 \%$ des témoins apparait comme trop faible.

\section{d) Essai en pots}

Afin de détailler et de contrôler les résultats du dispositif de terrain, un essai en pots a été réalisé en pépinière avec un sol d'une lande très voisine de celle où était implanté l'essai principal, mais qui s'est révélé à l'analyse nettement plus riche en potassium. Le dispositif était un factoriel $2^{4}$ combinant les éléments N,P. K. Ca ${ }^{1}$. Les résultats ont été les suivants sur la pousse des 2 années :

- effet extrêmement significatif et positif du phosphore (test $F 30$ fois supérieur au seuil de signification à $1 \%$ o)

- effet significatif à $1 \%$ du calcium : le léger effet positif du calcium dans l'essai de terrain, mais non significatif (tableau 9), a donc été confirmé et mis statistiquement en évidence;

- interaction $\mathrm{N} \times \mathrm{P}$ positive et significative à $1 \%$;

- interactions $\mathrm{P} \times \mathrm{K}$ et $\mathrm{P} \times \mathrm{Ca}$ positives et significatives à $1 \%$.

L'effet de l'azote est done nul et n'apparait qu'en interaction. Si l'on excepte le cas du potassium dont l'effet nul dans l'expérience en pots est explicable par la plus grande richesse du sol, les résultats de l'essai de terrain, en particulier l'effet du phosphore, sont donc confirmés et explicités.

\section{3. - Essai de Grand-Grammont}

\section{a) Situation}

Il a été installé sur le plateau de Millevaches, à $700 \mathrm{~m}$ d'altitude, dans un sol brun acide humifère sur granite à 2 micas. La plantation avait été effectuée au printemps 1964 dans une lande à fougère, canche flexueuse et genêt dont certaines parties au moins ont dû être ancien- 


\section{TABLEAU 11}

Essai de Grand-Grammont - Pousses annuelles $(\mathrm{cm})$

et hauteur totale en $1970(\mathrm{~cm})$

\section{TABLE 11}

Grand-Grammant Fxperinent - Annual height increments $(\mathrm{cm})$ and total height $(\mathrm{cm})$ at the end of 1970. calculated $\mathrm{F}$ values and L.S.D.
$\begin{array}{ll}\text { F theoriques } & 5 \%: 1,84 \\ \text { (theoretical } \mathrm{F} \text { values) } & 1 \%: 2,35\end{array}$
$1 \mathrm{l} \%: 3,08$

\begin{tabular}{|c|c|c|c|c|c|c|c|c|c|c|c|c|}
\hline Traitements & & $\begin{array}{l}\text { Age (1) } \\
\text { Annèe }\end{array}$ & $\begin{array}{c}4 \\
1967\end{array}$ & $\begin{array}{c}5 \\
1968\end{array}$ & $\begin{array}{c}6 \\
1969\end{array}$ & $\begin{array}{c}7 \\
1970\end{array}$ & Traitements & $\begin{array}{l}\text { Age (1) } \\
\text { Année }\end{array}$ & $\begin{array}{c}4 \\
1967\end{array}$ & $\stackrel{5}{1968}$ & $\begin{array}{c}6 \\
1969\end{array}$ & $\begin{array}{c}7 \\
1970\end{array}$ \\
\hline & & H. tot. 70 & & & & & & H. tot. 70 & & & & \\
\hline $\begin{array}{l}\mathrm{T} \\
\mathrm{N} \\
\mathrm{P} \\
\mathrm{K} \\
\mathrm{Ca} \\
\mathrm{NP} \\
\mathrm{NCa} \\
\mathrm{NK}\end{array}$ & & $\begin{array}{l}148,5 \\
141,2 \\
172,5 \\
155,4 \\
152,5 \\
171,0 \\
149,6 \\
158,0\end{array}$ & $\begin{array}{l}17,4 \\
19,7 \\
21,0 \\
17,7 \\
20,0 \\
23,1 \\
20,8 \\
22,1\end{array}$ & $\begin{array}{l}19,5 \\
16,3 \\
23,3 \\
19,7 \\
21,6 \\
23,9 \\
18,4 \\
19,2\end{array}$ & $\begin{array}{l}32,7 \\
28,0 \\
39,7 \\
33,4 \\
32,0 \\
39,9 \\
31,9 \\
36,0\end{array}$ & $\begin{array}{l}40,8 \\
40,0 \\
50,2 \\
38,8 \\
41,0 \\
44,9 \\
37,8 \\
44,8\end{array}$ & $\begin{array}{l}\mathrm{PK} \\
\mathrm{PCa} \\
\mathrm{KCa} \\
\mathrm{NPK} \\
\mathrm{NPCa} \\
\mathrm{NKCa} \\
\mathrm{PKCa} \\
\mathrm{NPKCa}\end{array}$ & $\begin{array}{l}162,8 \\
184,6 \\
169,5 \\
174,6 \\
190,6 \\
144,2 \\
178,0 \\
185,0\end{array}$ & $\begin{array}{l}20,1 \\
22,4 \\
21,7 \\
21,1 \\
22,0 \\
20,9 \\
23,8 \\
22,9\end{array}$ & $\begin{array}{l}24,3 \\
26,6 \\
22,9 \\
26,1 \\
27,7 \\
17,6 \\
27,0 \\
26,3\end{array}$ & $\begin{array}{l}36,6 \\
42,4 \\
37,5 \\
42,9 \\
45,5 \\
29,6 \\
43,0 \\
44,2\end{array}$ & $\begin{array}{l}41,9 \\
52,6 \\
46,3 \\
46,6 \\
51,8 \\
38,2 \\
49,6 \\
51,5\end{array}$ \\
\hline $\begin{array}{l}\text { F calculé } \\
\text { Ppds } \\
\text { (L.S.D.) }\end{array}$ & $\begin{array}{l}5 \% \\
1 \% \\
1 \%\end{array}$ & & & & & & & $\begin{array}{r}7,24 \\
16,32 \\
21,70 \\
27,74\end{array}$ & $\begin{array}{l}2,26 \\
0,75 \\
1,00 \\
1,10\end{array}$ & $\begin{array}{l}9,25 \\
3,44 \\
4,57 \\
5,88\end{array}$ & $\begin{array}{l}7,20 \\
4,86 \\
7,79 \\
9,96\end{array}$ & $\begin{array}{r}3,55 \\
7,82 \\
10,40 \\
13,29\end{array}$ \\
\hline
\end{tabular}

(1) Depuis la plantation (after planting) 
nement cultivées. L'analyse montre une texture sablo-argileuse et une bonne fertilité : 0,35 m.e de $\mathrm{K}$ échangeable pour $100 \mathrm{~g}$ et $0,69 \%$ de $\mathrm{P}_{2} \mathrm{O}_{5}, 0,53 \mathrm{~m}$.e de $\mathrm{Ca}$, un $\mathrm{C} / \mathrm{N}$ de 16,6 et un $\mathrm{pH}$ nettement acide de 4,5 .

\section{b) Protocole}

L'essai de fertilisation n'a été créé qu'à l'automne 1966, sur une plantation qui faisait donc alors sa $3^{e}$ pousse. Il s'agit d'un factoriel $2^{4}$ à 5 répétitions, combinant les traitements élémentaires N P K Ca définis comme suit :

$\mathrm{P}$ : apport en surface autour de chaque plant, sans binage, de $40 \mathrm{~g}$ de superphosphate triple à $48 \%$ en septembre $1966\left(20 \mathrm{~g}\right.$ de $\left.\mathrm{P}_{2} \mathrm{O}_{5}\right)$;

$\mathrm{K}$ : apport dans les mêmes conditions de $40 \mathrm{~g}$ de sulfate de potasse $\left(20 \mathrm{~g}\right.$ de $\left.\mathrm{K}_{2} \mathrm{O}\right)$;

$\mathrm{Ca}: 50 \mathrm{~g}$ de chaux éteinte par plant, appliqués de la mème façon ( $30 \mathrm{~g}$ de $\mathrm{CaO}$ );

$\mathrm{N}: 3,5 \mathrm{~g}$ d'azote au printemps 1967 en surface, sous forme d'ammonitrate, 4,5 g au printemps 1968 (urée) et 4,5 g au printemps 1969 (ammonitrate).

\section{c) Résultats}

Les pousses annuelles en hauteur sont indiquées au tableau 11. L'effet de la fertilisation est toujours significatif à partir de 1967, s'amplifie en 1968 et 1969 et diminue en 1970; chaque année, les meilleurs traitements sont ceux qui associent $\mathrm{P}$ et $\mathrm{Ca}$, le gain de croissance maximal étant de $34 \%$ par rapport au témoin.

Le dépouillement complet sur la hauteur totale à la fin de 1970 fait apparaitre un effet positif significatif à $1 \%$ (et même bien supérieur) du phosphore, un effet positif à $1 \%$ du calcium, et une interaction NP positive et significative à $5 \%$. Les résultats sont donc presqu'identiques ả ceux de l'essai de Razès en pots.

\section{TABleau 12}

Essai de Grand-Grammont ; analyses foliaires ( $\%$ de la matière sèche)

TABLE 12

Grand-Grammont Experiment : dutrient concentrations in one year needles $(\%$ of D. W.)

\begin{tabular}{|c|c|c|c|c|c|c|}
\hline Annce & $\begin{array}{l}\text { Age } \\
\text { (i) }\end{array}$ & Traitements & $\mathrm{N}$ & $\mathrm{P}_{2} \mathrm{O}_{6}$ & K & $\mathrm{Ca}$ \\
\hline $\begin{array}{l}1968 \\
1969 \\
1970\end{array}$ & $\begin{array}{l}5 \\
6 \\
7\end{array}$ & $\begin{array}{l}\mathrm{T} \\
\mathrm{T} \\
\mathrm{T} \\
\mathrm{Ca} \\
\mathrm{PCa} \\
\mathrm{NP} \\
\text { NPCa } \\
\text { NPKCa } \\
\text { PKCa }\end{array}$ & $\begin{array}{l}1,68 \\
1,88 \\
1,76 \\
1,94 \\
1,69 \\
1,83 \\
1,92 \\
1,87 \\
1,83\end{array}$ & $\begin{array}{l}0,31 \\
0,35 \\
0,41 \\
0,45 \\
0,49 \\
0,42 \\
0,56 \\
0,37 \\
0,41\end{array}$ & $\begin{array}{l}0,71 \\
0,79 \\
0,60 \\
0,63 \\
0,67 \\
0,66 \\
0,78 \\
0,70 \\
0,68\end{array}$ & $\begin{array}{l}0,54 \\
0,52 \\
0,67 \\
0,59 \\
0,50 \\
0,59 \\
0,55 \\
0,62 \\
0,56\end{array}$ \\
\hline
\end{tabular}

(1) Depuis la plantation (after planting)

Les analyses foliaires (tableau n $\mathrm{n}^{\circ} 12$ ) montrent une déficience nette en phosphore en 1968, alors que le niveau de la nutrition en $\mathrm{N}, \mathrm{K}$ et Ca est bon. La nutrition en phosphore 
s'améliore dans les témoins en 1969 sans toutefois que l'effet de cet élément sur la croissance s'atténue. En 1970, l'alimentation en phosphore s'est encore améliorée dans les témoins : la teneur des aiguilles y atteint $0,41 \%$ alors qu'elle fluctue entre 0,37 et $0,56 \%$ dans les meilleurs traitements ( $\mathrm{PCa}, \mathrm{NPCa}, \mathrm{NPKCa}, \mathrm{PKCa}$ ). On peut done penser quel'effet de la fertilisation est en voie d'atténuation, comme le confirme d'ailleurs la valeur du test $\mathrm{F}$.

\section{4. - Essai en pots sur le sol de Saint-Sétiers}

Il s'agit également d'un sol brun acide de lande à callune, très humifère (15\% de matière organique sur $20 \mathrm{~cm}$ ), sur granite à 2 micas, très proche de celui de Razès. Il a cependant un $\mathrm{C} / \mathrm{N}$ plus bas (16), une teneur plus élevée en calcium échangeable $(1,4 \mathrm{~m}$.e pour $100 \mathrm{~g}) \mathrm{et}$ en potassium $(0,52 \mathrm{~m}, \mathrm{e})$, et est plus pauvre en $\mathrm{P}_{2} \mathrm{O}_{5}(0,15 \%$ ), sans cependant que ce chiffre apparaisse comme très bas.

L'horizon A a été prélevé sur place, ainsi qu'une faible épaisseur de I'horizon (B), et le sol reconstitué dans des pots à la pépinière d'Amance près de Nancy. On a fertilisé les pots suivant un système factoriel $2^{4}$ identique à celui de Razès, en combinant les éléments $\mathrm{N}, \mathrm{P}, \mathrm{K}, \mathrm{Ca}$ suivant les traitements élémentaires suivants :

P : $9,6 \mathrm{~g}$. de $\mathrm{P}_{2} \mathrm{O}_{5}$ par pot de $10 \mathrm{dm}^{3}$ environ, sous forme de superphosphate triple, mélangé dans tout 1 horizon $A_{1}$ plusieurs semaines avant la plantation;

$\mathrm{Ca}: 20 \mathrm{~g}$ de $\mathrm{CaO}$ ( $30 \mathrm{~g}$ de chaux éteinte);

$\mathrm{K}: 3,5 \mathrm{~g}$ de $\mathrm{K}_{2} \mathrm{O}$ et $\mathrm{I}, 15 \mathrm{~g}$ de $\mathrm{MgO}$ sous forme de Patentkali;

$\mathrm{N}: 0,8 \mathrm{~g}$ d'azote en mai $1969,1 \mathrm{~g}$ en avril 1970 et $1,6 \mathrm{~g}$ en mai 1970 , sous forme d'ammonitrate.

Chacun des 16 traitements de l'expérience a été appliqué à 5 pots dans chacun desquels on a repiqué, en avril 1969, 10 semis de 1 an d'Épicéa; en 1970, on n'en a laissé subsister que 3 choisis comme ayant la hauteur la plus proche de la moyenne du traitement correspondant, calculée à la fin de 1969 .

Les hauteurs totales ont été mesurées fin 1970, donc sur des plants repiqués $1+2$. Les plants des meilleurs traitements atteignaient une hauteur moyenne de $24,5 \mathrm{~cm}$ contre 13,9 pour les témoins. Nous ne donnerons que les principaux résultats de l'interprétation factorielle :

- effet positif extrêmement important de $\mathrm{P}$ (test $\mathrm{F} 10$ fois supérieur au $\mathrm{F}$ théorique à $1 \%$;

- effet positif et significatif à 1\% de Ca mais dû surtout à l'effet du calcium sur les plants qui n'ont pas reçu de phosphore:

- interaction $\mathrm{N} \times \mathrm{P}$ positive et presque significative à $1 \%$;

- interaction $\mathrm{P} \times$ Ca négative, significative à $1 \%$, et sans doute dûe au blocage par la chaux d'une partie du phosphore.

Les analyses foliaires de quelques traitements sont données au tableau 13. Elles montrent nettement la sous-nutrition en phosphore du témoin et des plants fertilisés avec $\mathrm{N}$ seul ou Ca seul, mais son excellente alimentation en azote, alimentation cependant fragile qui baisse radicalement dès qu'on apporte du phosphore seul et du calcium seul. Elles montrent aussi la synergie de $\mathrm{N}$ et $\mathrm{P}$ qui explique l'interaction de ces deux éléments (le niveau de nutrition en $\mathrm{P}_{2} \mathrm{O}_{5}$ est nettement plus élevé pour le traitement NP que pour le traitement $\mathbf{P}$ seul). Enfin, les traitements NPK et NPCa qui fournissent les meilleurs résultats 
TABLEAU 13

Essai en pofs de St-Sétiers - Hanteur totale après 2 ans $(\mathrm{cm})$ ef analyses foliaires

(\% de la matière sèche)

TABLE 13

St-Sétiers pots Experiment - total height after two years and nutrient concentrations in one year needles (\% of D.W.)

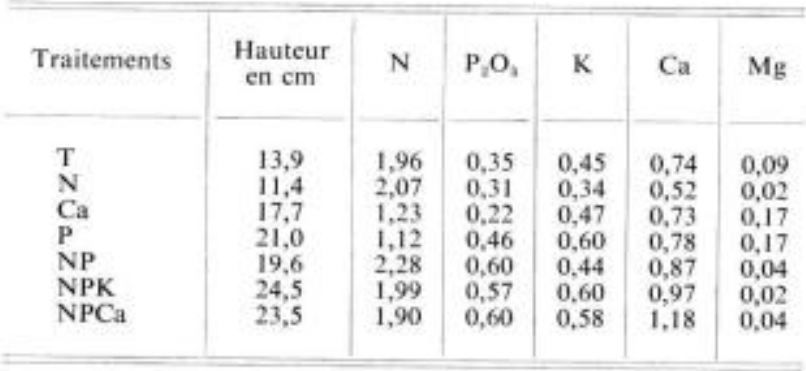

donnent une idée de l'optimum de composition foliaire, à l'exception du magnésium qui est probablement à un niveau trop faible.

La même expérience a été répétée deux fois sur deux provenances d'Épicéa différentes, Gérardmer et Haut-Doubs : les résultats sont les mêmes pour les deux,

\section{5. - Conclusion}

Seule l'expérience de St-Apollinaire dans le Rhône n'a donné aucun résultat. Mais si l'on considère les deux essais de terrain du Limousin (Grand-Grammont et Razès), complétés par les essais en pots de Razès et de St-Sétiers, on arrive à une grande unité dans les résultats de la fertilisation de I'Épicéa sur les sols bruns acides humifères développés sur granites à 2 micas. Le phosphore joue un rôle essentiel, le calcium a un effet positif mais assez faible qui n'apparait que dans les dispositifs factoriels très complets. L'azote n'a aucun rôle direct mais son interaction avec le phosphore est positive. Le potassium semble n'avoir d'effet que de manière exceptionnelle. L'interaction entre $\mathrm{P}$ et Ca est tantôt positive (essai en pots de Razès), tantôt nulle (Grand-Grammont), tantôt négative (St-Sétiers), mais on remarque que ces trois sols, rangés ainsi dans le sens d'une interaction $\mathrm{P} \times$ Ca décroissante, le sont aussi dans le sens d'une teneur en calcium échangeable croissante $(0,10$ m.e à Razès, 0,53 à Grand-Grammont et 1,4 à St-Sétiers). On remarque également que l'effet positif du potassium à Razès correspond ả la plus basse teneur des trois sols en $\mathrm{K}$ échangeable $(0,15 \mathrm{~m}$.e à Razès, 0,35 à Grand-Grammont, 0,52 à St-Sétiers).

Mais, en ce qui concerne le phosphore, l'analyse des sols conduit à des résultats curieux : la plupart des sols ont des teneurs en $\mathrm{P}_{2} \mathrm{O}_{5}$ assimilable trè3 supérieures $(0,15$ à $1 \%$ ) à celle que nous considérons habituellement comme un seuil de probabilité de l'effet de la fertilisation $(0,05 \%$ environ). Les sols humifères du Massif Central se comportent donc, sur ce plan, de manière tout à fait anormale et nous l'avions déjà fait remarquer dans une publication antérieure (BONNEAU et al., 1970). GACHON était, avant nous, parvenu à la même conclusion dans le domaine agricole pour les sols volcaniques humifères de la région de Clermont-Ferrand. 
L'effet de la fertilisation dans les premières années de la plantation est important (34 à $100 \%$ et se constate même sur les landes à genêt et fougère considérées par les praticiens comme très favorables à l'Épicéa. On ne peut, cependant, hasarder aucun pronostic sur la durée de cet effet, ni sur son incidence sur la production en volume.

\section{III. - NUTRITION DES JEUNES PLANTATIONS D'ÉPICÉA DANS LE MASSIF CENTRAL, PARTICULIÊREMENT EN LIMOUSIN}

Si l'on compare les analyses foliaires des essais d'altitude sur granite (contrat De Chalus, Arzenc-de-Randon, Ygrands) avec ceux de moyenne altitude (Razès, Grand-Grammont, St-Sétiers), on se rend compte que les premiers souffrent essentiellement d'une terrible carence azotée. Les résultats de la fertilisation le confirment largement. Même sur rochemère plus riche (Lac de Guéry), la nutrition azotée est loin d'être optimale. Le jaunissement caractéristique des jeunes plantations d'altitude est si généralisé et si bien connu des praticiens qu'il est inutile de démontrer davantage cette carence par des analyses foliaires supplémentaires. La figure 1 illustre nettement cette carence.

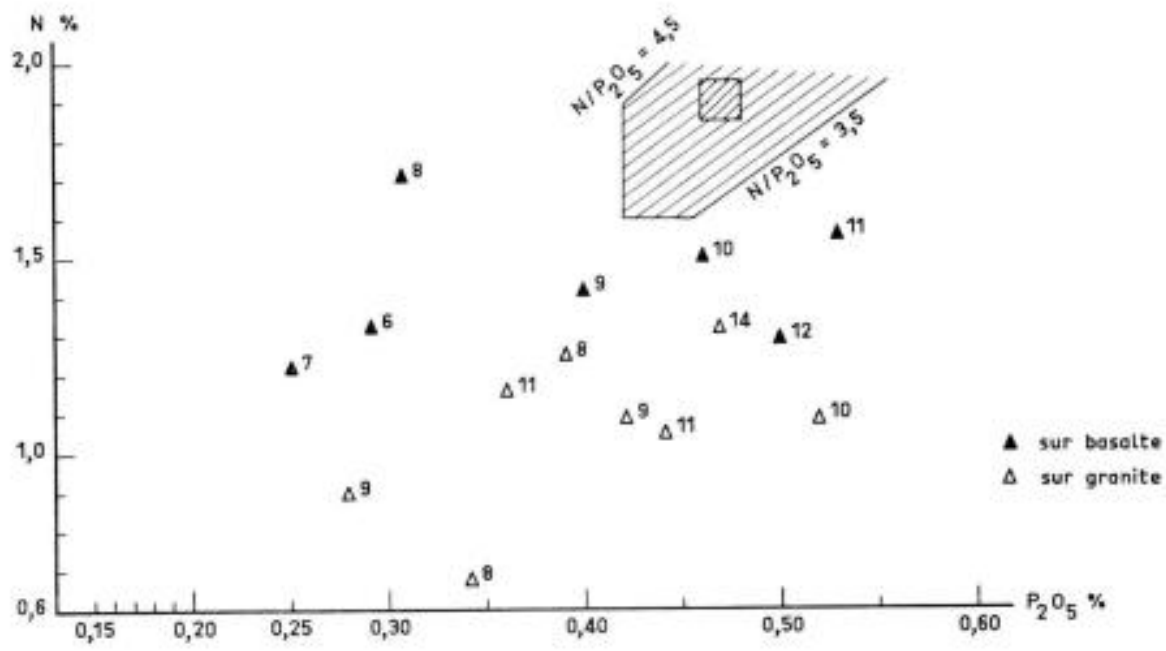

F1G. 1. - Nutrition en $\mathrm{P}_{8} \mathrm{O}_{3}$ et $\mathrm{N}$ des plantations de haute altitude (en $\%$ de matière sèche) - en hachures serrées : domaine de mutrition optimale

- en hachures lâches : mutrition suboptimale mais encore convenable.

L'äge total (y compris pépinière) est indiqué à côté du point représentatif

FIG. 1. - Nutrition in $\mathrm{N}$ and $\mathrm{P}_{3} \mathrm{O}_{5}$ of spruce plantations in great altitude sites $(\%$ of D. W.) - dense hatching : optimum of nutrition

- loose hatching : suboptimal but still satisfactory natrition

Total age is indicated near the representative point (including nursery time)

Par contre, à moyenne altitude, sur le plateau de Millevaches notamment, après parfois une crise passagère d'un an ou deux, les plantations se comportent généralement de manière assez satisfaisante et une investigation plus large n'est pas inutile pour se rendre compte 
si les résultats obtenus dans nos quelques essais de fertilisation sont généralisables. C'est pourquoi, depuis 3 ans, quelques jeunes plantations de 2 à 8 ans ont fait l'objet d'analyses foliaires répétées. Mais avant de discuter des résultats, il est indispensable de définir, à la lumière des essais de fertilisation et de données bibliographiques, un optimum de composition foliaire.

\section{1. - Optimum de composition foliaire}

L'optimum de nutrition peut varier notablement avec l'âge. Les 2 meilleurs traitements de l'essai en pots de St-Sétiers peuvent fournir, pour des épicéas de 3 ans, une composition optimale (tableau 13). HOFFMANN et INGESTADT ont fourni également des chiffres provenant d'essais, soit en pépinière, soit en solution nutritive. En combinant ces valeurs, d'ailleurs assez proches, nous arrivons à l'optimum indiquẻ au tableau 14.

TABLEAU 14

Analyses foliaires de plasieurs plantations de bonne croissance et optima de nutrition proposés

TABLE 14

Natrient concentrations in several good growing plantations and proposed optima of concentration

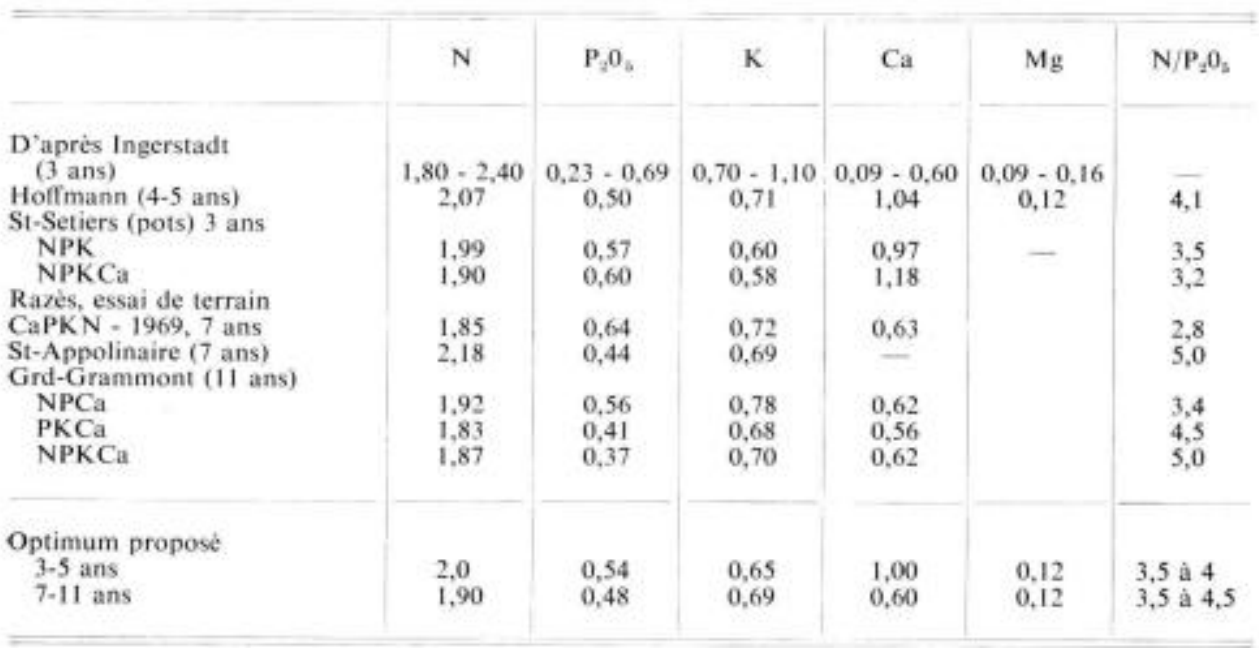

Pour des épicéas plus âgés, nous disposons des analyses du traitement CaPKN de Razès en 1969 et 1970 ( 7 et 8 ans y compris l'élevage en pépinière), de celles des traitements NPCa, PKCa, NPKCa de Grand-Grammont en 1970 (11 ans) et d'analyses des témoins de St-Apollinaire en 1965 (7 ans), essai où la fertilisation n'a pas eu d'effet (pour cet essai le calcium, dont les résultats étaient suspects, a été éliminé). En combinant ces diverses valeurs, nous pouvons nous fixer également des ordres de grandeur vraisemblables des optima pour un âge de 7 à 11 ans. Nous ne hasarderons aucun chiffre personnel pour le magnésium, le procédé colorimétrique de dosage que nous employons n'étant pas très exact. D'après les données bibliographiques, il doit se situer à $0,12 \%$ environ. 
Les chiffres donnés au tableau 14, valables pour de jeunes plantations, sont notablement plus élevés que les optima généralement admis pour des arbres adultes.

Le tableau 14 donne également les valeurs correspondantes du rapport $\mathrm{N} / \mathrm{P}_{2} \mathrm{O}_{5}$ qui a une certaine valeur de diagnostic, encore qu'il puisse varier dans d'assez larges proportions aux approches de l'optimum (HoLsTENER JORGENSEN, 1971).

\section{2. - Analyses foliaires de quelques plantations du Limousin et leur interprétation}

20 stations ont été examinées ${ }^{1}$, soit sur lande à callune, soit sur lande à fougère, à des altitudes très voisines; toutes sauf une, Valéoux (sur granite à biotite), sont situées sur granite à 2 micas.

Dans chacune, les pousses annuelles de 100 plants ont été mesurées et les prélèvements foliaires effectués sur 10 plants dont la pousse annuelle s'écartait au maximum de $10 \%$ de la moyenne de 100 plants. Les 10 prélèvements ont été mélangés pour constituer l'échantillon moyen soumis à l'analyse.

\subsection{1. - Landes à callune (tableau 15 et figure 2).}

Les plus jeunes plantations examinées ( 2 ans) ont toutes une croissance faible, mais leur nutrition est très différente suivant le travail du sol. Dans la plantation en potets (Chaumeil), la nutrition en azote et en phosphore est extrêmement déficiente, le feuillage extrêmement jaune des jeunes plants correspondant essentiellement à la carence en azote. La nutrition en potassium elle-même, sans atteindre la carence véritable, reste insuffisante. Cette situation n'est cependant que provisoire : à partir de la $3^{\circ}$ année, en même temps que le feuillage reverdit, la nutrition azotée s'améliore et peut devenir très proche de l'optimum (Chaumeil) ou rester insuffisante (Pradines). La nutrition en phosphore reste nettement trop faible.

Au contraire, lorsque le sol a été bien travaillé avec élimination de la callune sur une bande assez large autour des plants par décapage, passage de rotavator ou de root-rake, la nutrition azotée est d'emblée trẻs bonne dès la $2^{\mathrm{e}}$ année et dépasse même l'optimum. Il en est de même pour la nutrition en potassium. Par contre, l'alimentation en $\mathrm{P}_{2} \mathrm{O}_{5}$ reste notoirement insuffisante dans tous les cas, atteignant même parfois le seuil de carence de $0,23 \%$ (Péret - Bel-air).

Lorsque les plantations vieillissent, l'effet du travail du sol s'estompe progressivement, la callune se réinstalle et la nutrition azotée commence à se dégrader. Ceci apparait déjả clairement pour les jeunes reboisements de Cleydat et Razès. A partir de la $4^{e}$ année de plantation, on observe presque dans tous les cas (sauf Chaumeil et St-Sétiers) des teneurs en azote nettement insuffisantes et mème parfois très mauvaises (La Sagne, Neypoux, Larfeuil, Le Bourg). La figure 2 illustre bien cette pèjoration, avec l'âge, de la nutrition azotée.

Le rapport $\mathrm{N} / \mathrm{P}_{2} \mathrm{O}_{5}$ reste cependant la plupart du temps supérieur à l'optimum car la nutrition en $\mathrm{P}_{2} \mathrm{O}_{5}$ est, dans tous les cas, beaucoup trop faible.

La nutrition en calcium semble insuffisante pour les jeunes plants, mais s'améliore au contraire pour les plantations plus âgées.

1. Nous remercions M. CouNIL, Ingénieur des travaux des Eaux et Foréts à la D.D.A. de Corrèze de nous avoir indiqué la plupart d'entre elles. 
TABLEAU 15

Composition foliaire de queloues plantations d'Épicéa sur lande à callune dans l'Ouest du Massif Central. Pour la signification des symboles, voir légende du tableau 16

TABLE 15

Nutrient contents of one year needles of several sprace plantations on calluna heathland in West of the Massif Central $(\%$ of D.W.) (For the notes, see on the table 16 )

\begin{tabular}{|c|c|c|c|c|c|c|c|c|c|c|c|}
\hline \multirow{2}{*}{ Plantation } & \multirow{2}{*}{$\begin{array}{l}\text { Travail } \\
\text { du Sol }\end{array}$} & \multirow{2}{*}{ Année } & \multirow{2}{*}{$\begin{array}{c}\text { Age depuis } \\
\text { la plantation } \\
\text { (a) }\end{array}$} & \multirow{2}{*}{$\begin{array}{l}\text { Age total } \\
\text { y compris } \\
\text { pépiniere } \\
\text { (b) }\end{array}$} & \multirow{2}{*}{$\begin{array}{l}\text { Pousse } \\
\text { annuelle } \\
\text { (cm) } \\
\text { (c) }\end{array}$} & \multicolumn{5}{|c|}{ Composition foliaire $\%$} & \multirow{2}{*}{$\mathrm{P}_{2} \mathrm{O}_{5}$} \\
\hline & & & & & & N & $\mathrm{P}_{2} \mathrm{O}_{5}$ & K & $\mathrm{Ca}$ & $\mathrm{Mg}$ & \\
\hline Chaumeil & $\mathbf{P}$ & $\begin{array}{l}1968 \\
1969 \\
1970 \\
\end{array}$ & $\begin{array}{l}2 \\
3 \\
4 \\
\end{array}$ & $\begin{array}{l}6 \\
7 \\
8 \\
\end{array}$ & $\begin{array}{r}2,6 \\
11,4 \\
24,6\end{array}$ & $\begin{array}{l}0,69 \\
1,81 \\
1,98 \\
\end{array}$ & $\begin{array}{l}0,30 \\
0,41 \\
0,48\end{array}$ & $\begin{array}{l}0,58 \\
0,72 \\
0,57\end{array}$ & $\begin{array}{l}0,47 \\
0,65 \\
0,70\end{array}$ & $\begin{array}{l}0,12 \\
0,12 \\
0,22\end{array}$ & $\begin{array}{l}2,3 \\
4,4 \\
4,1 \\
\end{array}$ \\
\hline St-Sétiers & RS & $\begin{array}{l}1968 \\
1969 \\
1970\end{array}$ & $\begin{array}{l}2 \\
3 \\
4 \\
\end{array}$ & $\begin{array}{l}6 \\
7 \\
8\end{array}$ & $\begin{array}{r}1,4 \\
16,6 \\
21,4 \\
\end{array}$ & $\begin{array}{l}2,39 \\
2,00 \\
1,91\end{array}$ & $\begin{array}{l}0,41 \\
0,33 \\
0,33\end{array}$ & $\begin{array}{l}0,64 \\
0,67 \\
0,49\end{array}$ & $\begin{array}{l}0,52 \\
0,45 \\
0,68\end{array}$ & $\begin{array}{l}0,13 \\
0,09 \\
0,11\end{array}$ & $\begin{array}{l}5,8 \\
6,0 \\
5,8\end{array}$ \\
\hline Cleydat & RS & $\begin{array}{l}1968 \\
1969 \\
1970 \\
\end{array}$ & $\begin{array}{l}2 \\
3 \\
4 \\
\end{array}$ & $\begin{array}{l}6 \\
7 \\
8 \\
\end{array}$ & $\begin{array}{r}1,5 \\
13,1 \\
32,8\end{array}$ & $\begin{array}{l}2,25 \\
2,16 \\
1,47\end{array}$ & $\begin{array}{l}0,34 \\
0,27 \\
0,42\end{array}$ & $\begin{array}{l}0,66 \\
0,67 \\
0,54\end{array}$ & $\begin{array}{l}0,45 \\
0,54 \\
0,69\end{array}$ & $\begin{array}{l}0,14 \\
0,05 \\
0,13\end{array}$ & $\begin{array}{l}6,6 \\
8,0 \\
3,5\end{array}$ \\
\hline Razès & ES & $\begin{array}{l}1968 \\
1969 \\
1970 \\
\end{array}$ & $\begin{array}{l}2 \\
3 \\
4\end{array}$ & $\begin{array}{l}6 \\
7 \\
8\end{array}$ & $\begin{array}{r}2,9 \\
24,5 \\
25,2\end{array}$ & $\begin{array}{l}2,45 \\
1,83 \\
1,60\end{array}$ & $\begin{array}{l}0,43 \\
0,35 \\
0,33\end{array}$ & $\begin{array}{l}0,67 \\
0,61 \\
0,53\end{array}$ & $\begin{array}{l}0,26 \\
0,14 \\
0,24\end{array}$ & $\begin{array}{l}0,14 \\
0,10 \\
0,10\end{array}$ & $\begin{array}{l}5,7 \\
5,2 \\
4,9\end{array}$ \\
\hline Peret Bel-air & RS & $\begin{array}{l}1969 \\
1970\end{array}$ & $\begin{array}{l}3 \\
4 \\
\end{array}$ & $\begin{array}{l}7 \\
8\end{array}$ & $\begin{array}{r}13,1 \\
8,6\end{array}$ & $\begin{array}{l}1,75 \\
1,34\end{array}$ & $\begin{array}{l}0,18 \\
0,22\end{array}$ & $\begin{array}{l}0,60 \\
0,58\end{array}$ & $\begin{array}{l}0,41 \\
0,60\end{array}$ & $\begin{array}{l}0,08 \\
0,13 \\
\end{array}$ & $\begin{array}{l}9,7 \\
6,1\end{array}$ \\
\hline Pradines & $\mathrm{P}$ & $\begin{array}{l}1969 \\
1970 \\
\end{array}$ & $\begin{array}{l}4 \\
5 \\
\end{array}$ & $\begin{array}{l}8 \\
9 \\
\end{array}$ & $\begin{array}{r}6,9 \\
11,2 \\
\end{array}$ & $\begin{array}{l}1,64 \\
1,56\end{array}$ & $\begin{array}{l}0,30 \\
0,33\end{array}$ & $\begin{array}{l}0,66 \\
0,55\end{array}$ & $\begin{array}{l}0,50 \\
0,68\end{array}$ & $\begin{array}{l}0,15 \\
0,12\end{array}$ & $\begin{array}{l}5,5 \\
4,7\end{array}$ \\
\hline Davignacq & DS & $\begin{array}{l}1968 \\
1969 \\
1970 \\
\end{array}$ & $\begin{array}{l}4 \\
5 \\
6\end{array}$ & $\begin{array}{r}8 \\
9 \\
10 \\
\end{array}$ & $\begin{array}{r}8,0 \\
18,0 \\
24,5\end{array}$ & $\begin{array}{l}1,99 \\
1,40 \\
1.60\end{array}$ & $\begin{array}{l}0,37 \\
0,33 \\
0,36\end{array}$ & $\begin{array}{l}0,72 \\
0,69 \\
0,65\end{array}$ & $\begin{array}{l}0,45 \\
0,43 \\
0,51\end{array}$ & $\begin{array}{l}0,14 \\
0,04 \\
0,11\end{array}$ & $\begin{array}{l}5,4 \\
4,2 \\
4,4\end{array}$ \\
\hline La Sagne & RS & $\begin{array}{l}1969 \\
1970 \\
\end{array}$ & $\begin{array}{l}5 \\
6\end{array}$ & $\begin{array}{r}9 \\
10 \\
\end{array}$ & $\begin{array}{l}16,7 \\
26,4 \\
\end{array}$ & $\begin{array}{l}1.17 \\
1.15\end{array}$ & $\begin{array}{l}0,22 \\
0,24\end{array}$ & $\begin{array}{l}0,70 \\
0,54\end{array}$ & $\begin{array}{l}0,38 \\
0,68\end{array}$ & $\begin{array}{l}0,08 \\
0,11\end{array}$ & $\begin{array}{l}5,3 \\
4,8 \\
\end{array}$ \\
\hline Neypoux & $\mathbf{P}$ & $\begin{array}{l}1969 \\
1970 \\
\end{array}$ & $\begin{array}{l}5 \\
6\end{array}$ & $\begin{array}{r}9 \\
10 \\
\end{array}$ & $\begin{array}{l}20,7 \\
22,4\end{array}$ & $\begin{array}{l}1,51 \\
1,27\end{array}$ & $\begin{array}{l}0,31 \\
0,28\end{array}$ & $\begin{array}{l}0,69 \\
0,53\end{array}$ & $\begin{array}{l}0,41 \\
0,61\end{array}$ & $\begin{array}{l}0,10 \\
0,09\end{array}$ & $\begin{array}{l}4,9 \\
4,5\end{array}$ \\
\hline Le Bourg & DS & $\begin{array}{l}1969 \\
1970 \\
\end{array}$ & $\begin{array}{l}7 \\
8 \\
\end{array}$ & $\begin{array}{l}11 \\
12\end{array}$ & $\begin{array}{l}30,1 \\
23,9\end{array}$ & $\begin{array}{l}1,11 \\
1,20\end{array}$ & $\begin{array}{l}0,25 \\
0,34\end{array}$ & $\begin{array}{l}0,87 \\
0,57\end{array}$ & $\begin{array}{l}0,42 \\
0,69\end{array}$ & $\begin{array}{l}0,10 \\
0,12\end{array}$ & $\begin{array}{l}4,4 \\
3,5 \\
\end{array}$ \\
\hline Larfeuil & $\mathrm{R}$ & $\begin{array}{l}1968 \\
1969 \\
1970\end{array}$ & $\begin{array}{l}7 \\
8 \\
9\end{array}$ & $\begin{array}{l}11 \\
12 \\
13\end{array}$ & $\begin{array}{l}16,7 \\
18,8 \\
16,2\end{array}$ & $\begin{array}{l}1,50 \\
1,10 \\
1,04\end{array}$ & $\begin{array}{l}0,25 \\
0,19 \\
0,20\end{array}$ & $\begin{array}{l}0,64 \\
0,68 \\
0,61\end{array}$ & $\begin{array}{l}0,47 \\
0,33 \\
0,62\end{array}$ & $\begin{array}{l}0,09 \\
0,10 \\
0,10\end{array}$ & $\begin{array}{l}6,0 \\
5,8 \\
5,2\end{array}$ \\
\hline
\end{tabular}


Ainsi, les résultats des essais de fertilisation dont nous disposons, soit au champ, soit en pots, et qui mettent en lumière un important effet du phosphore, sont donc à cet égard en parfaite concordance avec la nutrition des diverses plantations examinées. La fertilisation phosphatée des jeunes reboisements sur lande à callune parait done indispensable et généralisable. La faible action de la fertilisation azotée s'accorde également bien avec les résultats de l'enquête sur les plantations les plus jeunes. Mais l'insuffisance d'alimentation en azote, mise en évidence à partir de la $5^{\mathrm{e}}$ année après la plantation, indique que la fertilisation azotée qui a été appliquée dans nos expériences pendant 3 ans après la plantation, ne l'a pas été au moment le plus opportun, ce qui explique son effet faible ou nul. Mais une application à partir de 5 ans devrait se révéler efficace.

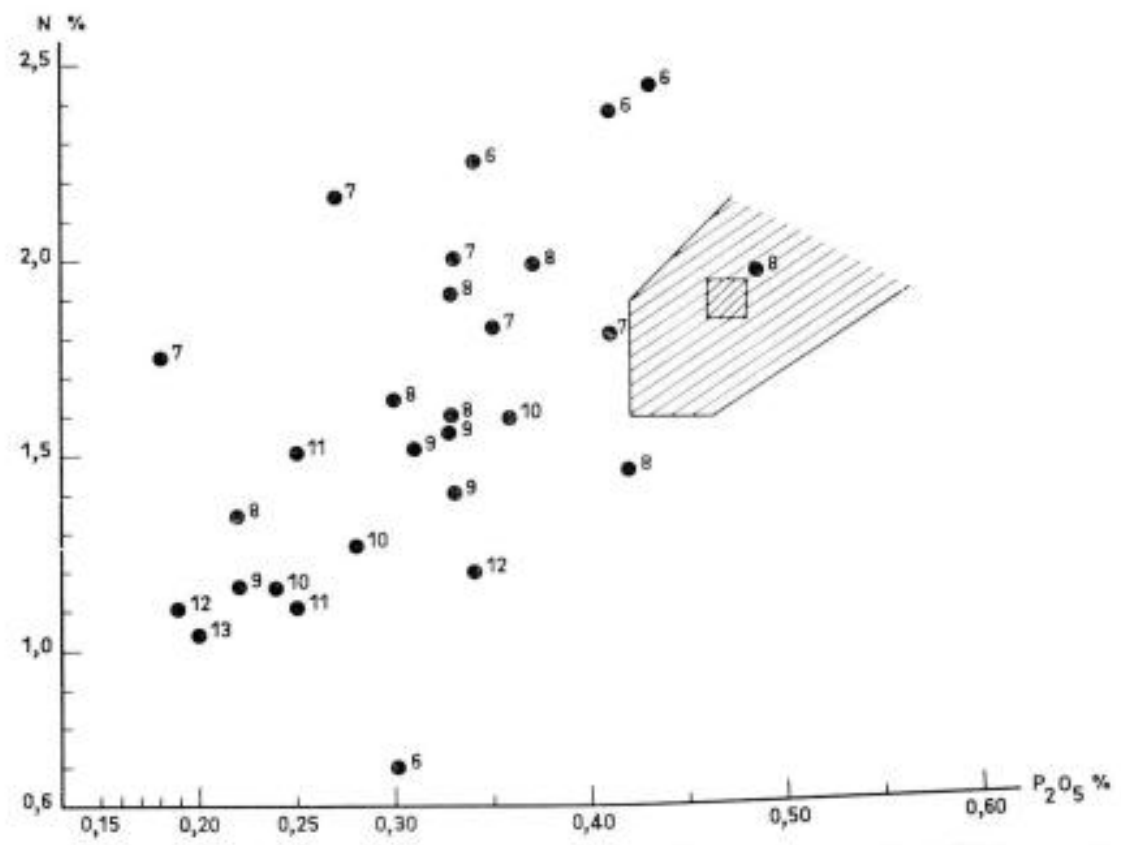

Fio, 2. - Nutrition en $\mathrm{N}$ et $\mathrm{P}_{2} \mathrm{O}_{5}$ des plantations sur lande à callune à moyenne alritude (\% de la matière sèche) hachures : Voir graphique l

FiG. 2. - Nutrition in $\mathrm{N}$ and $\mathrm{P}_{2} \mathrm{O}_{3}$ of sprace plantation on heath-sites at middle altirude $(\%$ of D. W.) haching : see figsure l

\subsection{2. - Landes à fougère. (tableau 16 et figure 3 ).}

Dans la plupart des cas, les teneurs en azote des aiguilles sont proches de l'optimum; elles ne s'en éloignent notablement que dans le cas où la plantation a été faite en potets sans travail du sol (Chaumeil, Chamberet, Pradines) encore ce défaut de nutrition n'est-il pas continu et $n$ 'intervient-il que certaines années, différentes d'une plantation à l'autre.

La teneur en $\mathrm{P}_{2} \mathrm{O}_{5}$ des aiguilles est également assez bonne et ne descend de manière notable au-dessous de l'optimum qu'exceptionnellement (Chaumeil en 1970, Grand-Grammont). Notre essai de Grand-Grammont se situe donc dans des conditions relativement défavorables au sein de l'ensemble des reboisements sur lande à fougère.

La nutrition en potassium ne descend que rarement et de peu au-dessous de l'optimum, de même que la nutrition en calcium. 
TABLEAU 16

Composition foliaire de quelques plantations d'Épicéa sur lande à fougère dans rOnest du Massif Central

Travail du sol - P : plantation en potets; RS : rotavator et sous-solage; ES ; enlèvement de la callune au root-rake et sous-solage; DS : décapage et sous-solage.

TABLE 16

Nutrient contents of one year needles of several spruce plantations on braken land in West of the Massif Central $(\%$ of D.W.)

Modality of soil preparation - $\mathbf{P}$ : planting in holes without soil preparation; RS; rotary land breaking

and sub-soil ploughing; ES; carryng off the vegetation with root-rake and sub-soil ploughing; DS : stripping and sub-soil ploughing.

Column a) age of plantation; b) total age including nursery time ; c) annual growth (cm).

\begin{tabular}{|c|c|c|c|c|c|c|c|c|c|c|c|}
\hline \multirow{2}{*}{ Plantation } & \multirow{2}{*}{$\begin{array}{l}\text { Travail } \\
\text { du Sol }\end{array}$} & \multirow{2}{*}{ Année } & \multirow{2}{*}{$\begin{array}{c}\text { Age depuis } \\
\text { la plantation } \\
\text { (a) }\end{array}$} & \multirow{2}{*}{$\begin{array}{l}\text { Age total } \\
\text { y eompris } \\
\text { pépinière } \\
\text { (b) }\end{array}$} & \multirow{2}{*}{$\begin{array}{l}\text { Pousse } \\
\text { annuelle } \\
(\mathrm{cm}) \\
\text { (c) }\end{array}$} & \multicolumn{5}{|c|}{ Composition foliaire $\%$} & \multirow{2}{*}{$\begin{array}{c}\mathrm{N} / \\
\mathrm{P}_{2} \mathrm{O}_{5}\end{array}$} \\
\hline & & & & & & $\mathrm{N}$ & $\mathrm{P}_{2} \mathrm{O}_{5}$ & K & $\mathrm{Ca}$ & $\mathrm{Mg}$ & \\
\hline Chaumeil & $\mathbf{P}$ & $\begin{array}{l}1968 \\
1969 \\
1970\end{array}$ & $\begin{array}{l}2 \\
3 \\
4\end{array}$ & $\begin{array}{l}6 \\
7 \\
8\end{array}$ & $\begin{array}{r}2,8 \\
16,5 \\
17,2\end{array}$ & $\begin{array}{l}1,80 \\
1,68 \\
1,50\end{array}$ & $\begin{array}{l}0,45 \\
0,45 \\
0,34\end{array}$ & $\begin{array}{l}0,72 \\
0,83 \\
0,60\end{array}$ & $\begin{array}{l}0,50 \\
0,49 \\
0.54\end{array}$ & $\begin{array}{l}0,23 \\
0,12 \\
0,22\end{array}$ & $\begin{array}{l}4,0 \\
3,7 \\
4,4\end{array}$ \\
\hline Cleydat & DS & $\begin{array}{l}1968 \\
1969 \\
1970 \\
\end{array}$ & $\begin{array}{l}2 \\
3 \\
4\end{array}$ & $\begin{array}{l}6 \\
7 \\
8\end{array}$ & $\begin{array}{r}2,8 \\
21,5 \\
23,6\end{array}$ & $\begin{array}{l}1,82 \\
1,94 \\
1,84\end{array}$ & $\begin{array}{l}0,47 \\
0,54 \\
0,53\end{array}$ & $\begin{array}{l}0,80 \\
0,82 \\
0,64\end{array}$ & $\begin{array}{l}0,52 \\
0,54 \\
0,71\end{array}$ & $\begin{array}{l}0,23 \\
0,16 \\
0,13\end{array}$ & $\begin{array}{l}3,9 \\
3,6 \\
3,5\end{array}$ \\
\hline Chamberet & $\mathrm{P}$ & $\begin{array}{l}1969 \\
1970\end{array}$ & $\begin{array}{l}3 \\
4 \\
\end{array}$ & $\begin{array}{l}7 \\
8 \\
\end{array}$ & $\begin{array}{l}26,3 \\
23,5\end{array}$ & $\begin{array}{l}1,55 \\
1,71\end{array}$ & $\begin{array}{l}0,44 \\
0,46\end{array}$ & $\begin{array}{l}0.77 \\
0,62 \\
\end{array}$ & $\begin{array}{l}0,34 \\
0,60\end{array}$ & $\begin{array}{l}0,10 \\
0,22\end{array}$ & $\begin{array}{l}3,5 \\
3,7\end{array}$ \\
\hline $\mathrm{G}^{\mathrm{a}}-\mathrm{Grammont}$ & RS & $\begin{array}{l}1968 \\
1969 \\
1970 \\
\end{array}$ & $\begin{array}{l}4 \\
5 \\
6\end{array}$ & $\begin{array}{r}8 \\
9 \\
10 \\
\end{array}$ & $\begin{array}{l}20,4 \\
35,0 \\
41,9 \\
\end{array}$ & $\begin{array}{l}1,68 \\
1,88 \\
1,76\end{array}$ & $\begin{array}{l}0,31 \\
0,35 \\
0,41\end{array}$ & $\begin{array}{l}0,71 \\
0,79 \\
0,60\end{array}$ & $\begin{array}{l}0,54 \\
0,52 \\
0,67\end{array}$ & $\begin{array}{l}0,09 \\
0,10 \\
0,10\end{array}$ & $\begin{array}{l}5,4 \\
5,4 \\
4,3\end{array}$ \\
\hline Pradines & $\mathrm{P}$ & $\begin{array}{l}1969 \\
1970 \\
\end{array}$ & $\begin{array}{l}4 \\
5\end{array}$ & $\begin{array}{l}8 \\
9 \\
\end{array}$ & $\begin{array}{l}20,1 \\
25,6\end{array}$ & $\begin{array}{l}1,88 \\
1,37\end{array}$ & $\begin{array}{l}0,63 \\
0,45\end{array}$ & $\begin{array}{l}0,88 \\
0,60 \\
\end{array}$ & $\begin{array}{l}0,60 \\
0,63\end{array}$ & $\begin{array}{l}0,15 \\
0,13 \\
\end{array}$ & $\begin{array}{l}3,0 \\
3,1\end{array}$ \\
\hline Davignacy & DS & $\begin{array}{l}1969 \\
1970 \\
\end{array}$ & $\begin{array}{l}5 \\
6\end{array}$ & $\begin{array}{r}9 \\
10 \\
\end{array}$ & $\begin{array}{l}24,4 \\
36,7 \\
\end{array}$ & $\begin{array}{l}2,10 \\
1,90\end{array}$ & $\begin{array}{l}0,60 \\
0,46\end{array}$ & $\begin{array}{l}0,88 \\
0,68\end{array}$ & $\begin{array}{l}0,53 \\
0,62 \\
\end{array}$ & $\begin{array}{l}0,26 \\
0,17 \\
\end{array}$ & $\begin{array}{l}3,5 \\
4,1\end{array}$ \\
\hline La Sagne & RS & $\begin{array}{l}1969 \\
1970 \\
\end{array}$ & $\begin{array}{l}5 \\
6\end{array}$ & $\begin{array}{r}9 \\
10\end{array}$ & $\begin{array}{l}28,3 \\
37,3\end{array}$ & $\begin{array}{l}2,05 \\
1,87\end{array}$ & $\begin{array}{l}0,42 \\
0,46\end{array}$ & $\begin{array}{l}0,80 \\
0,58 \\
\end{array}$ & $\begin{array}{l}0,36 \\
0,62 \\
\end{array}$ & $\begin{array}{l}0,10 \\
0,11\end{array}$ & $\begin{array}{l}4,9 \\
4,1\end{array}$ \\
\hline Valeoux & DS & $\begin{array}{l}1969 \\
1970 \\
\end{array}$ & $\begin{array}{l}6 \\
7\end{array}$ & $\begin{array}{l}10 \\
11\end{array}$ & $\begin{array}{l}35,2 \\
39,7\end{array}$ & $\begin{array}{l}1,99 \\
1,79\end{array}$ & $\begin{array}{l}0,48 \\
0,44\end{array}$ & $\begin{array}{l}0,89 \\
0,55\end{array}$ & $\begin{array}{l}0,38 \\
0,55\end{array}$ & $\begin{array}{l}0,11 \\
0.10\end{array}$ & $\begin{array}{l}4,1 \\
4,1\end{array}$ \\
\hline Les Géants & $\mathrm{P}$ & $\begin{array}{l}1969 \\
1970\end{array}$ & $\begin{array}{l}6 \\
7\end{array}$ & $\begin{array}{l}10 \\
11\end{array}$ & $\begin{array}{l}31,8 \\
49,4\end{array}$ & $\begin{array}{l}1,67 \\
1,93\end{array}$ & $\begin{array}{l}0,56 \\
0,71\end{array}$ & $\begin{array}{l}0,70 \\
0,71\end{array}$ & $\begin{array}{l}0,42 \\
0,68\end{array}$ & $\begin{array}{l}0,11 \\
0.22\end{array}$ & $\begin{array}{l}3,0 \\
2,7\end{array}$ \\
\hline
\end{tabular}


Dans ces conditions écologiques, il ne semble donc pas opportun, dans l'état actuel de nos connaissances, de généraliser la fertilisation des jeunes plantations.

La figure 4 montre que, parallèlement à la nutrition, la croissance est très différente dans les landes à callune et dans les landes à fougère : dans les premières, la pousse annuelle, après avoir augmenté régulièrement jusqu'à la $4^{e}$ année, se stabilise à des valeurs médiocres tandis que, dans la seconde, elle augmente constamment, du moins pendant la durée à laquelle se sont limitées nos observations.

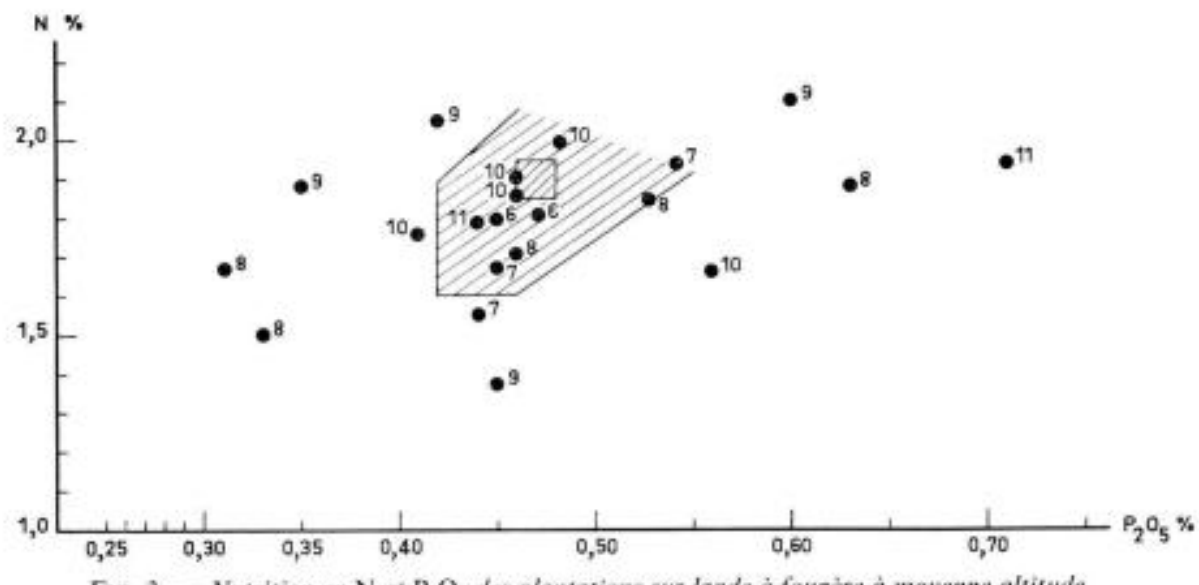

FiG. 3, - Nutrition en $\mathrm{N}$ et $\mathrm{P}_{2} \mathrm{O}_{3}$ des plantations sur lande à fougère à moyenne aluitude hachures : Voir graphique $I$

FIc. 3. - Nutrition in $\mathrm{N}$ and $\mathrm{P}_{2} \mathrm{O}_{5}$ of sprace plantations on braken-sine at middle altitude haching : See figure $l$

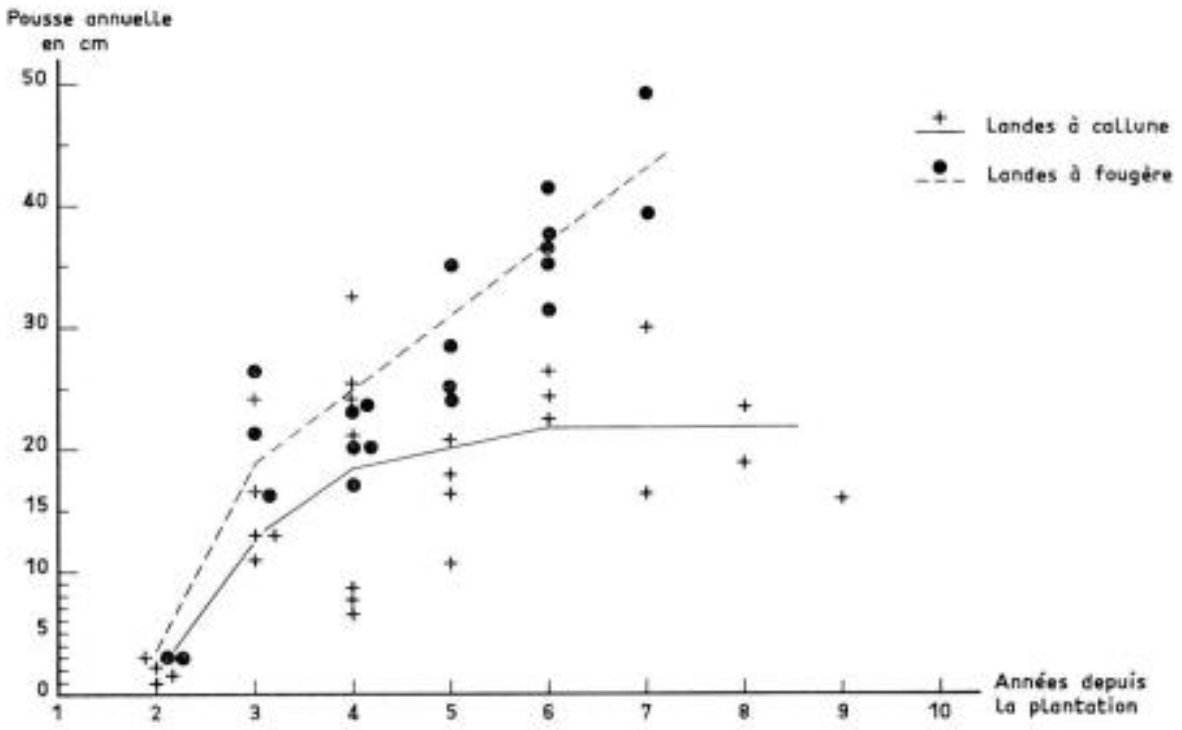

FiG. 4. - Pousses annuelles des plantations d'Epicéa dans Touest du Massif Central - trait plein : landes à callune - pointillé : landes à fougère

F1G. 4. - Annual length growth of spruce plantations in West of the Massif Central - uninterrupted line : hearh-sites - interrupted line : braken-land 


\section{CONCLUSIONS}

Parmi les différentes stations du Massif Central qui ont donné lieu à des investigations suffisantes, soit par expérimentation directe, soit par simple enquête de nutrition, nous pouvons faire schématiquement quatre catégories qui apparaissent clairement sur les graphiques 1 à 3 .

\section{1. - Stations d'altitude sur roche-mère riche (basalte) (figure 1)}

La nutrition azotée n'atteint pas l'optimum mais reste relativement convenable; les engrais azotés n'ont qu'une action faible et sporadique. La nutrition phosphatée, d'abord mauvaise, s'améliore progressivement. Une fertilisation à base de phosphore, calcium et potassium (oủ le calcium et le phosphore sont vraisemblablement les éléments les plus actifs) améliore fortement la croissance des quatre premières années; son effet diminue progressivement ensuite tout en restant très hautement significatif. Cette fertilisation agit favorablement, de manière indirecte, sur la nutrition azotée.

\section{2. - Stations d'altitude sur roche-mère granitique (figure 1)}

La carence en azote est extrême. Une fertilisation azotée est indispensable mais son action est considérablement renforcée, suivant les cas, par l'apport de phosphore ou de calcium. L'apport de calcium, pour être efficace, doit être important et permettre la saturation a $50 \%$ au moins du complexe absorbant. Dans la pratique, on peut recommander l'apport de $1000 \mathrm{~kg}$ de chaux éteinte par ha et de $200 \mathrm{~kg}$ de phosphate naturel ou de scories concentrés sur les lignes de plantation.

Même les apports d'engrais azotés restent impuissants à porter la nutrition en azote à son optimum; ils ne l'améliorent que légèrement pendant les années où ils sont pratiqués et deux ou trois ans après, mais leur action disparaît ensuite. II faudrait que ces apports soient très abondants et renouvelés très fréquemment, tous les ans ou tous les deux ans.

Un apport d'humus brut de pessière améliore de manière durable la croissance et on assiste, en même temps, à une modification de la mycorhization des plants par rapport aux témoins. On ne peut cependant affirmer que le changement de type des mycorhizes soit la cause directe de l'augmentation de croissance.

\section{3. - Landes à callune à moyenne altitude (figure 2)}

Une insuffisance de nutrition en phosphore est très généralement observée et une fertilisation phosphatée améliore considérablement la croissance en hauteur. La nutrition potassique semble quelquefois un peu inférieure à l'optimum. L'alimentation en azote, d'abord optimale pendant les 3 ou 4 premiẻres années si le sol a été convenablement travaillé, se dégrade rapidement ensuite à mesure que la callune envahit à nouveau le terrain. Une fertilisation azotée, dont l'effet semble nul ou faible dans nos expérimentations encore récentes, devrait avoir un effet positif à partir de la $5^{e}$ année environ après la plantation.

\section{4. - Landes à fougère à moyenne altitude (figure 3)}

Elles constituent des stations beaucoup plus fertiles ce qui est bien connu empiriquement des reboiseurs. La nutrition en azote et potassium est bonne et la nutrition phosphatée 
ne se révèle insuffisante que dans un petit nombre de situations. Malgré le succès d'une expérimentation dans l'un de ces cas, il ne semble pas opportun de conseiller une fertilisation systématique dans l'ensemble des landes à fougère.

On doit s'interroger sur la rentabilité des diverses formules de fertilisation conseillées. Les plantations expérimentales étant très jeunes, il serait vain et dangereux de supputer les accroissements de production en volume à attendre de l'application des engrais.

On ne peut, pour le moment, que discuter des économies possibles de dégagement.

A haute altitude, sur un sol décapé et sous-solé, les dégagements ả prévoir sont à peu près nuls dans tous les cas, que le sol ait été ou non fertilisé. L'intérêt pratique de la fertilisation ne peut donc résulter que d'une meilleure installation et d'une meilleure vigueur des plants; compte tenu de la croissance de toute manière très lente, c'est peut-être plus une satisfaction psychologique qu'un gain réel.

A moyenne altitude, dans les landes à callune, après travail du sol, les dégagements à prévoir sont faibles dans le cas d'une réinstallation de callune, végétation rase qui ne concurrence guère la partic aćrienne des plants.

La fertilisation n'a donc que peu de chances de permettre une économie d'entretien. Son intérêt ne pourra résulter que d'un accroissement éventuel du volume produit.

Dans les landes à fougère, les dégagements sont toujours nécessaires. La fertilisation n'apporte, nous l'avons vu, qu'un faible gain relatif de croissance; cependant, pour les moins fertiles de ces stations, les résultats de l'essai de Grand-Grammont permettent de calculer qu'en fin de $6^{\circ}$ année, la hauteur totale des plants avait atteint $75 \mathrm{~cm}$ environ pour les témoins et $96 \mathrm{~cm}$ pour les plants fertilisés. En général, on admet que les entretiens peuvent cesser lorsque les plants ont $1 \mathrm{~m}$ de haut. La fertilisation permet donc, dans des cas semblables, de gagner un dégagement, ce qui rembourse plus de la moitié de la somme investie en apport d'engrais (coût d'un dégagement : $150 \mathrm{~F} /$ ha pour un coût de fertilisation de $250 \mathrm{~F}$ environ).

Il est probable que ce gain sur les dégagements s'étendrait aussi aux meilleures stations des landes à callune où l'on assiste fréquemment, aprẻs travail du sol, à un envahissement de fougère et où des dégagements peuvent devenir nécessaires.

Recu pour publication en novembre 1971 .

\section{SUMMARY}

CONTRIBUTION TO THE KNOWLEDGE OF NUTRITION AND FERTILIZATION

OF PLANTATIONS OF NORWAY SPRUCE IN MASSIF CENTRAL.

By fertilization experiments and nutrition studies (foliar analysis), we can range young Norway Spruce plantations of the Massif Central in four categories with regard to the site.

\section{a. - Great altitude sites on rich parent rock (hasalte) : tables 1, 2; fig. I}

At first phosphorus nutrition is poor but it becomes better after four years. Fertilizing with $\mathrm{Ca}, \mathrm{P}, \mathrm{K}$ at planting time increases length growth of about $80 \%$ during four years and $25 \%$ only after. Nitrogen nutrition is not very poor but does not reach the optimum. However, $\mathrm{N}$ fertilizers have no effect because $\mathrm{N}$ uptake is improved by CaPK fertilizers. 
b. - Great altitude sites on acid parent rock (granit) : tables $3,4,5,6,7,8$; fig. 1

Nitrogen deficiency is very heavy. $\mathrm{N}$ fertilizing is indispensable but its effect becomes stronger with application of $\mathrm{P}$ or $\mathrm{Ca}$. Ca supply must be large enough to make a $50 \%$ saturation of the exchange capacity. For practical use, it is recommanded to bring $1000 \mathrm{~kg}$ of Ca $(\mathrm{OH})_{2}$ and $200 \mathrm{~kg}$ of basic slags, concentrated on scraped strips before plantation.

Although $\mathrm{N}$ fertilizers have a good effect on growth, $\mathrm{N}$ concentration in needles remains very low and the effect of $\mathrm{N}$ fertilizers does not continue longer than three years after the last application.

Application of $100 \mathrm{~g}$ of spruce raw humus in plantation holes gives durable growth improvement and change in type of mycorrhization. But it is not certain that this change is the cause of the faster growth.

\title{
c. - Middle altitude Calluna hearhlands (table 15, fig. 2)
}

In these sites, phosphorus nutrition is deficient and P fertilizing gives a large growth improvement which sometimes reachs $100 \%$. Nitrogen nutrition is good during a few years when plantation was made with important soil cultivation but becomes worse and worse while the soil is again covered by calluna. $\mathrm{N}$ fertilizer probably could have a good effect from the sixth year after planting.

\section{d) Middle altitude brakenlands (table 16; fig. 3)}

They are fertile sites where $\mathrm{N}$ and $\mathrm{K}$ nutrition is good. Only in a few eases, $\mathrm{P}$ nutrition is unsatisfactory. Although in one experiment growth was improved by $\mathbf{P}$ fertilizers, systematic fertilizing of young plantations does not seem necessary in these sites.

\section{ZUSAMMENFASSUNG}

\section{BEITRAG ZUR UNTERSUCHUNG DER ERNÄHRUNG UND DER DÜNGUNG}

\author{
DER FICHTENPFLANZUNGEN IM ZENTRAL MASSIV
}

Düngungversuche und Nadelanalysen in Fichtenpflanzungen im Zentrum und Western des Zentral Massives ermöglichten die folgenden wichtigsten Standorttypen zu unterscheiden.

$$
\text { a. - Hochlagen auf reichen Muttergesteinen (Basalt) - Tab. I u. } 2 \text { - Abb. I }
$$

In den ersten Jahren, ist die Phosphorernährung unzureichend; nach vier Jahren, wird sie jedoch zufriedenstellend. Nach einer PKCa Düngung bei der Pflanzung erhöht sich der Zuwachs um $80 \%$, er fallt jedoch nach vier Jahren auf $25 \%$ ab. Obwohl die Stickstoffernährung das Optimum nicht erreicht, zeigt eine zuzätzliche N Düngung keinen Erfolg.

$$
\text { b. - Hochlagen auf armen Muttergestein (Granit) - Tab. 3, 4, 5, 6, 7, } 8 \text { - Abb. I }
$$

Es besteht ein ausgeprägter Stickstoffmangel und eine N Düngung ist unarlässlich; ihre Wirkung wird durch eine zuzätliche $\mathrm{P}$ oder $\mathrm{Ca}$ Düngung verstärkt. Für die Praxis, ist die Ausbringung von Thomasphosphat $(200 \mathrm{~kg} / \mathrm{ha})$ und Branntkalk (1000 kg/ha) auf den Pflugstreifen zu empfehlen. Obgleich der Stickstoff einen guten Erfolg zeigt, dauert seine Wirkung jedoch nur bis zum dritten Jahr nach der letzten Ausbringung.

Durch Ermischung von $100 \mathrm{~g}$ Fichtenrohhumus in der Pflanzloch, wird der Zuwachs um $25 \%$ bis $50 \%$ dauerhaft erhöht; zugleich, ist eine Veränderung der Mycorhizatypen zu beobachten. Ob diese Änderung der Mycorhizabildung die Ursache der Zuwachsförderung ist, bleibt jedoch nicht erklärt.

\section{c) Mitrellagen Calluna-heiden}

Die Plianzungen leiden an starkem Phosphormangel und der Zuwachs wird, manchmal bis zu $100 \%$, durch eine Phosphatdüngung erhöht. Wenn der Boden gut bearbeitet worden ist, erreicht der Stickstoffgehalt der Nadeln während der ersten Jahre einen hohen Wert; wenn die Calluna wiederum nachwächst, verschlechtert sich aber die Stickstoffernährung. Nach dem sechsten Jahre hätte wahrscheinlich eine $\mathrm{N}$ Düngung einen guten Effekt. 


\section{d) Mittellagen Adlerfarnstandorte}

Es sind fruchtbare mit einer guten $\mathrm{N}$ und $\mathrm{K}$ Ernährung. Nur in einigen Făllen, bleibt die $\mathrm{P}$ Ernährung unter dem Optimum. Trotz der guten Ergebnisse eines Düngungsversuches, dürfte eine systematische Düngung auf diesen Standorten nicht zu empfehlen sein.

\section{RÉFÉRENCES BIBLIOGRAPHIQUES}

Bonneau M., 1965. Premiers résultats d'essais de fertilisation de l'Épicéa à haute altitude dans le Massif Central. Ann. sci. For. $X X I I(3)$.

Bonneau M., 1967. Minéralisation de l'azote de deux sols des Hautes-Vosges : allure du phénomène et tentatives d'amélioration. Science du Sol (l).

Duchaufour Ph., 1965. Un cas de carence azotée de l'Épicèa décelée par le diagnostic foliaire. R.F.F. (2).

GACHON, 1966. Phosphore isotopiquement diluable et pouvoir fixateur des sols en relation avec la crois. sance des plantes. C.R. Acad. Agric., $n^{\circ} / 5$.

HOFFManN F., 1967. Untersuchungen zür Düngung und Emahrung von Fichtenverschulungen. Arch. Forstw., 16 (2),

Holstener-Jorgiensev, 1971, Rapport au XVe Congres de I'I.U.F.R.O. Gainesville (U.S.A.).

Marquestaut J., 1970. La croissance de l'Épicéa commun dans les landes à callune du Massif Central. Bulletin A.F.O.C.E.L., 1970. 